\title{
The integral monodromy of hyperelliptic and trielliptic curves
}

\author{
Jeffrey D. Achter \& Rachel Pries *
}

\begin{abstract}
We compute the $\mathbb{Z} / \ell$ and $\mathbb{Z}_{\ell}$ monodromy of every irreducible component of the moduli spaces of hyperelliptic and trielliptic curves. In particular, we provide a proof that the $\mathbb{Z} / \ell$ monodromy of the moduli space of hyperelliptic curves of genus $g$ is the symplectic group $\mathrm{Sp}_{2 g}(\mathbb{Z} / \ell)$. We prove that the $\mathbb{Z} / \ell$ monodromy of the moduli space of trielliptic curves with signature $(r, s)$ is the special unitary group $\mathrm{SU}_{(r, s)}\left(\mathbb{Z} / \ell \otimes \mathbb{Z}\left[\zeta_{3}\right]\right)$.
\end{abstract}

MSC 11G18, 14D05, 14H40

keywords monodromy, hyperelliptic, trigonal, moduli, Jacobian

\section{Introduction}

If $C \rightarrow S$ is a relative smooth proper curve of genus $g \geq 1$ over an irreducible base, then the $\ell$-torsion of the relative Jacobian of $C$ encodes important information about the family. Suppose $\ell$ is invertible on $S$, and let $s \in S$ be a geometric point. The fundamental group $\pi_{1}(S, s)$ acts linearly on the fiber $\operatorname{Pic}^{0}(C)[\ell]_{s} \cong(\mathbb{Z} / \ell)^{2 g}$, and one can consider the mod- $\ell$ monodromy representation associated to $C$ :

$$
\rho_{C \rightarrow S, \ell}: \pi_{1}(S, s) \rightarrow \operatorname{Aut}\left(\operatorname{Pic}^{0}(C)[\ell]_{s}\right) \cong G_{2 g}(\mathbb{Z} / \ell) .
$$

Let $\mathrm{M}_{\ell}(C \rightarrow S)$, or simply $\mathrm{M}_{\ell}(S)$, be the image of this representation. If a primitive $\ell^{\text {th }}$ root of unity is defined globally on $S$, then $\operatorname{Pic}^{0}(C)[\ell]_{S}$ is equipped with a skew-symmetric form $\langle\cdot, \cdot\rangle$ and $\mathrm{M}_{\ell}(C \rightarrow S) \subseteq \mathrm{Sp}\left(\operatorname{Pic}^{0}(C)[\ell]_{S},\langle\cdot, \cdot\rangle\right) \cong \operatorname{Sp}_{2 g}(\mathbb{Z} / \ell)$. If $C \rightarrow S$ is a sufficiently general family of curves, then $\mathrm{M}_{\ell}(C \rightarrow S) \cong \mathrm{Sp}_{2 g}(\mathbb{Z} / \ell)[8]$.

In this paper, we compute $\mathrm{M}_{\ell}(S)$ when $S$ is an irreducible component of the moduli space of hyperelliptic or trielliptic curves and $C \rightarrow S$ is the tautological curve. The first result implies that there is no restriction on the monodromy group in the hyperelliptic case other than that it preserve the symplectic pairing. As a trielliptic curve is a $\mathbb{Z} / 3$-cover of a genus zero curve, the $\mathbb{Z} / 3$-action constrains the monodromy group to lie in a unitary group associated to $\mathbb{Z}\left[\zeta_{3}\right]$. The second result implies that this is the only additional restriction in the trielliptic case.

Theorem 3.4 Let $\ell$ be an odd prime, and let $k$ be an algebraically closed field in which $2 \ell$ is invertible. For $g \geq 1, \mathrm{M}_{\ell}\left(\mathcal{H}_{g} \otimes k\right) \cong \mathrm{Sp}_{2 g}(\mathbb{Z} / \ell)$.

*The second author was partially supported by NSF grant DMS-04-00461. 
Theorem 3.9 Let $\ell \geq 5$ be prime, and let $k$ be an algebraically closed field in which $3 \ell$ is invertible. Let $\mathcal{T}^{\bar{\gamma}}$ be any component of the moduli space of trielliptic curves of genus $g \geq 3$. Then $\mathrm{M}_{\ell}\left(\mathcal{T}^{\gamma} \otimes k\right) \cong \mathrm{SG}_{\left(r_{\gamma}, s_{\gamma}\right)}(\mathbb{Z} / \ell)$ (where the latter is a unitary group defined in (3.4)).

We also prove that the $\ell$-adic monodromy group is $\operatorname{Sp}_{2 g}\left(\mathbb{Z}_{\ell}\right)$ in the situation of Theorem 3.4 and is $\mathrm{SG}_{\left(r_{\gamma}, s_{\gamma}\right)}\left(\mathbb{Z}_{\ell}\right)$ in the situation of Theorem 3.9

Theorem 3.4 is an unpublished result of J.K. Yu and has already been used multiple times in the literature. In [7], Chavdarov assumes this result to show that the numerator of the zeta function of the typical hyperelliptic curve over a finite field is irreducible. Kowalski also uses this result in a similar fashion [20]. The first author used Theorem 3.4 to prove a conjecture of Friedman and Washington on class groups of quadratic function fields [2].

There are other results in the literature which are similar to Theorem 3.4 but which are not quite strong enough for the applications above. A'Campo [1, Th. 1] computes the topological monodromy of $\mathcal{H}_{g} \otimes \mathbb{C}$. On the arithmetic side, the $\mathbb{Q}_{\ell}$, as opposed to $\mathbb{Z}_{\ell}$, monodromy of $\mathcal{H}_{g}$ is computed in [16, 10.1.16]. Combined with a theorem of Larsen on compatible families of representations [22, 3.17], this shows that the mod- $\ell$ monodromy group of $\mathcal{H}_{g}$ is maximal for a set of primes $\ell$ of density one (as opposed to for all $\ell \geq 3$ ).

There are results on $\mathbb{Q}_{\ell}$-monodromy of cyclic covers of the projective line of arbitrary degree, e.g., [15. Sec. 7.9]. Also, in [12, 5.5], the authors prove that the projective representation $\mathbb{P} \rho_{C \rightarrow S, \ell}$ is surjective for many families of cyclic covers of the projective line. Due to a combinatorial hypothesis, their theorem does not apply to $\mathcal{H}_{g}$ and applies to at most one component of the moduli space of trielliptic curves for each genus, see Remark 2.7 See also work of Zarhin, e.g., [28].

As an application, for all $p \geq 5$, we show using [6] that there exist hyperelliptic and trielliptic curves of every genus (and signature) defined over $\overline{\mathbb{F}}_{p}$ whose Jacobians are absolutely simple. In contrast with the applications above, these corollaries do not use the full strength of our results. Related work can be found in [14] where the authors produce curves with absolutely simple Jacobians over $\mathbb{F}_{p}$ under the restriction $g \leq 3$.

Corollary 3.6 Let $p \neq 2$ and let $g \in \mathbb{N}$. Then there exists a smooth hyperelliptic curve of genus $g$ defined over $\overline{\mathbb{F}}_{p}$ whose Jacobian is absolutely simple.

Corollary 3.11 Let $p \neq 3$. Let $g \geq 3$ and let $(r, s)$ be a trielliptic signature for $g$ (Definition 2.9]. Then there exists a smooth trielliptic curve defined over $\overline{\mathbb{F}}_{p}$ with genus $g$ and signature $(r, s)$ whose Jacobian is absolutely simple.

Our proofs proceed by induction on the genus. The base cases for the hyperelliptic family rely on the fact that every curve of genus $g=1,2$ is hyperelliptic; the claim on monodromy follows from the analogous assertion about the monodromy of $\mathcal{M}_{g}$. The base case $g=3$ for the trielliptic family involves a comparison with a Shimura variety of PEL type, namely, the Picard modular variety. An important step is to show that the monodromy group does not change in the base cases when one adds a labeling of the ramification points to the moduli problem.

The inductive step is similar to the method used in [9] and uses the fact that families of smooth hyperelliptic (trielliptic) curves degenerate to trees of hyperelliptic (triellip- 
tic) curves of lower genus. The combinatorics of admissible degenerations require us to compute the monodromy exactly for the inductive step rather than up to isomorphism.

The inductive strategy using admissible degeneration developed here should work for other families of curves, especially for more general cyclic covers of the projective line. The difficulty is in the direct calculation of monodromy for the necessary base cases.

We thank C.-L. Chai, R. Hain, A.J. de Jong, E. Kani, and J. Kass.

\section{Moduli spaces of curves with $\mathbb{Z} / d$-action}

\subsection{Stable $\mathbb{Z} / d$-covers of a genus zero curve}

Let $G=\mathbb{Z} / d$ be a cyclic group of prime order $d$. Let $G^{\times}=G-\mathrm{id}_{G}$. Let $S$ be an irreducible scheme over Spec $\mathbb{Z}\left[1 / d, \zeta_{d}\right]$. Let $k$ be an algebraically closed field equipped with a map $\mathbb{Z}\left[1 / d, \zeta_{d}\right] \rightarrow k$

Let $\psi: C \rightarrow S$ be a semi-stable curve. In other words, $\psi$ is flat and proper and the geometric fibers of $C$ are connected, reduced curves whose only singularities are ordinary double points. If $s \in S$, let $C_{S}$ denote the fiber of $C$ over $s$. Let $\operatorname{Sing}_{S}(C)$ be the set of $z \in C$ for which $z$ is a singular point of the fiber $C_{\psi(z)}$.

A mark $\Xi$ on $C / S$ is a closed subscheme of $C-\operatorname{Sing}_{S}(C)$ which is finite and étale over $S$. The degree of $\Xi$ is the number of points in any geometric fiber of $\Xi \rightarrow S$. A marked semi-stable curve $(C / S, \Xi)$ is stably marked if every geometric fiber of $C$ satisfies the following condition: every irreducible component of genus zero has at least three points which are either in $\operatorname{Sing}_{S}(C)$ or on the mark $\Xi$.

Consider a $G$-action $\iota_{0}: G \hookrightarrow \operatorname{Aut}_{S}(C)$ on $C$. Denote the ramification locus of $C \rightarrow$ $C / \iota_{0}(G)$ by $R$, and the smooth ramification locus by $R_{\mathrm{sm}}=R-\left(R \cap \operatorname{Sing}_{S}(C)\right)$. We say that $\left(C / S, \iota_{0}\right)$ is a stable $G$-curve if $C / S$ is a semi-stable curve; if $\iota_{0}: G \hookrightarrow \operatorname{Aut}_{S}(C)$ is an action of $G$; if $R_{\mathrm{sm}}$ is a mark on $C / S$; and if $\left(C / S, R_{\mathrm{sm}}\right)$ is stably marked. We note that the definition implies that the dihedral nodes of [10, Def. 1.3] do not occur for $\left(C / S, \iota_{0}\right)$.

We say that a stable $G$-curve $\left(C / S, \iota_{0}\right)$ is admissible if the following conditions are satisfied for every geometric point $z \in R \cap \operatorname{Sing}_{S}(C)$. Let $C_{z, 1}$ and $C_{z, 2}$ denote the two components of the formal completion of $C_{\psi(z)}$ at $z$. First, $\iota_{0}(1)$ stabilizes each branch $C_{z, i}$; second, the characters of the action of $\iota_{0}$ on the tangent spaces of $C_{z, 1}$ and $C_{z, 2}$ at $z$ are inverses.

Throughout the paper, we suppose that $\left(C / S, \iota_{0}\right)$ is an admissible stable $G$-curve. We further assume throughout that $C / \iota_{0}(G)$ has arithmetic genus 0 . Then $C / \iota_{0}(G)$ is also a stably marked curve [10, Prop. 1.4]. The mark on $C / \iota_{0}(G)$ is the smooth branch locus $B_{\mathrm{sm}}$, which is the (reduced subscheme of) the image of $R_{\mathrm{sm}}$ under the morphism $C \rightarrow C / \iota_{0}(G)$. Let $r$ be the degree of $R_{\mathrm{sm}}$. By the Riemann-Hurwitz formula, the arithmetic genus of each fiber of $C$ is $g=1-d+r(d-1) / 2$.

Let $s$ be a geometric point of $S$ with residue field $k$ and let $a$ be a point of the fiber $R_{\mathrm{sm}, s}$. Then $G$ acts on the tangent space of $C_{s}$ at $a$ via a character $\chi_{a}: G \rightarrow k^{\times}$. In particular, there is a unique choice of $\gamma_{a} \in(\mathbb{Z} / d)^{\times}$so that $\chi_{a}(1)=\zeta_{d}^{\gamma_{a}}$. We say that $\gamma_{a}$ is the canonical generator of inertia at $a$. The inertia type of $\left(C / S, \iota_{0}\right)$ is the multiset $\left\{\gamma_{a}: a \in R_{\mathrm{sm}, s}\right\}$. It is independent of the choice of $s$. By Riemann's existence theorem, $\sum_{a \in R_{\mathrm{sm}, s}} \gamma_{a}=0 \in \mathbb{Z} / d$. 
We say that a mark $\Xi$ has a labeling if $\Xi$ is an ordered disjoint union of sections $S \rightarrow$ $C$. If $\Xi$ has degree $r$, we denote the labeling by $\eta:\{1, \ldots, r\} \rightarrow \Xi$. A labeling of an admissible stable $G$-curve $\left(C / S, \iota_{0}\right)$ is a labeling $\eta$ of $R_{\mathrm{sm}}$. There is an induced labeling $\eta_{0}:\{1, \ldots, r\} \rightarrow B_{\text {sm }}$.

If $\left(C / S, \iota_{0}, \eta\right)$ is a labeled $G$-curve, the class vector is the map of sets $\gamma:\{1, \ldots, r\} \rightarrow$ $G^{\times}$such that $\gamma(i)=\gamma_{\eta(i)}$. We frequently write $\gamma=(\gamma(1), \ldots, \gamma(r))$. If $\gamma$ is a class vector, we denote its inertia type by $\bar{\gamma}: G^{\times} \rightarrow \mathbb{Z}_{\geq 0}$ where $\bar{\gamma}(h)=\# \gamma^{-1}(h)$ for all $h \in G$.

\subsection{Moduli spaces}

We define moduli functors on the category of schemes over Spec $\mathbb{Z}\left[1 / d, \zeta_{d}\right]$ by describing their S-points:

$\overline{\mathcal{M}}_{G}$ parametrizes admissible stable $G$-curves $\left(C / S, \iota_{0}\right)$.

$\widetilde{\mathcal{M}}_{G}$ parametrizes labeled admissible stable G-curves $\left(C / S, \iota_{0}, \eta\right)$.

$\widetilde{\mathcal{M}}_{g, r}$ parametrizes triples $(C / S, \Xi, \eta)$ where $C / S$ is a semi-stable curve of genus $g, \Xi$ is a mark of degree $r$ on $C$ such that $(C / S, \Xi)$ is stably marked, and $\eta$ is a labeling of $\Xi$.

Each functor is represented by an algebraic stack, and we use the same letter to denote both a moduli functor and its representing stack. For each of these moduli spaces $\mathcal{M}$, we let $\mathcal{M}^{\circ}$ denote the open substack whose objects parametrize smooth curves of the appropriate type. To work with fibers of the structural map $\mathcal{M} \rightarrow \operatorname{Spec} \mathbb{Z}\left[1 / d, \zeta_{d}\right]$ we write $\mathcal{M} \otimes k$ for $\mathcal{M} \times_{\text {Spec } \mathbb{Z}\left[1 / d, \zeta_{d}\right]}$ Spec $k$.

Lemma 2.1. The moduli spaces $\widetilde{\mathcal{M}}_{G}$ and $\overline{\mathcal{M}}_{G}$ are smooth, proper Deligne-Mumford stacks over Spec $\mathbb{Z}\left[1 / d, \zeta_{d}\right]$. The subspaces $\widetilde{\mathcal{M}}_{G}^{\circ}$ and $\overline{\mathcal{M}}_{G}^{\circ}$ are open and dense in $\widetilde{\mathcal{M}}_{G}$ and $\overline{\mathcal{M}}_{G}$, respectively.

Proof. The moduli spaces $\widetilde{\mathcal{M}}_{G}$ and $\overline{\mathcal{M}}_{G}$ are algebraic stacks. Since the automorphism group scheme of a (labeled) admissible stable G-curve over an algebraically closed field is étale, $\overline{\mathcal{M}}_{G}$ and $\widetilde{\mathcal{M}}_{G}$ are Deligne-Mumford stacks [23, 8.1].

The local deformation problem for a semi-stable curve with $G$-action is formally smooth [10, 2.1], so $\overline{\mathcal{M}}_{G}$ is smooth. By [10, 2.3], the degree of the smooth ramification divisor of a stable admissible $G$-curve is locally constant, so $\widetilde{\mathcal{M}}_{G}$ is smooth, too.

We use the valuative criterion to show that these stacks are proper [23, 7.3]. Let $\mathcal{O}_{K}$ be a complete discrete valuation ring with field of fractions $K$, and suppose $\left(C, \iota_{0}\right) \in$ $\overline{\mathcal{M}}_{G}(K)$. By the stable reduction theorem, there exists a finite extension $K^{\prime} / K$ so that the curve $C$ extends as a stable curve to $\mathcal{O}_{K^{\prime}}$, as does the admissible $G$-action $\iota_{0}$. Possibly after a further extension of the base, one may blow up the special fiber of $C / \mathcal{O}_{K^{\prime}}$ to remove any dihedral nodes [10, p.195], so that no singular point of $C$ is in the closure of $R_{\mathrm{sm}}$ and the resulting curve is an admissible stable $G$-curve. This shows that $\overline{\mathcal{M}}_{G}$ is proper. After a finite base change, a labeling of $R_{\mathrm{sm}}$ also extends uniquely. Thus, $\widetilde{\mathcal{M}}_{G}$ is also proper.

The openness and density of $\widetilde{\mathcal{M}}_{G}^{\circ}$ in $\widetilde{\mathcal{M}}_{G}$ and of $\overline{\mathcal{M}}_{G}^{\circ}$ in $\overline{\mathcal{M}}_{G}$ follow from the fact that an admissible stable $G$-curve is equivariantly smoothable [10, 2.2]. 
Consequently, every connected component of $\overline{\mathcal{M}}_{G}$ or $\widetilde{\mathcal{M}}_{G}$ is irreducible.

Let $\gamma:\{1, \ldots, r\} \rightarrow(\mathbb{Z} / d)^{\times}$be a class vector of length $r=r(\gamma)$ for $G$. Let $g(\gamma):=$ $1-d+r(\gamma)(d-1) / 2$. Let $\widetilde{\mathcal{M}}_{G}^{\gamma}$ be the substack of $\widetilde{\mathcal{M}}_{G}$ for which $\left(C / S, \iota_{0}, \eta\right)$ has class vector $\gamma$. Let $\overline{\mathcal{M}}_{G}^{\bar{\gamma}}$ be the substack of $\overline{\mathcal{M}}_{G}$ for which $\left(C / S, \iota_{0}\right)$ has inertia type $\bar{\gamma}$.

Lemma 2.2. The moduli space $\widetilde{\mathcal{M}}_{G}^{\gamma}$ is irreducible.

Proof. Since $\widetilde{\mathcal{M}}_{G}$ is proper and smooth over Spec $\mathbb{Z}\left[1 / d, \zeta_{d}\right]$, it is sufficient to prove that $\widetilde{\mathcal{M}}_{G}^{\gamma} \otimes \mathbb{C}$ is irreducible [11, IV.5.10]. Since $\widetilde{\mathcal{M}}_{G}^{\gamma, \circ} \otimes \mathbb{C}$ is open and dense in $\widetilde{\mathcal{M}}_{G}^{\gamma} \otimes \mathbb{C}$, it suffices to prove that $\widetilde{\mathcal{M}}_{G}^{\gamma, \circ} \otimes \mathbb{C}$ is irreducible. Consider the functor $\beta_{\gamma}: \widetilde{\mathcal{M}}_{G}^{\gamma} \rightarrow \widetilde{\mathcal{M}}_{0, r(\gamma)}$. On $S$ points, this functor takes the isomorphism class of $\left(C, \iota_{0}, \eta\right)$ to the isomorphism class of $\left(C / \iota_{0}(G), B_{\mathrm{sm}}, \eta_{0}\right)$, where $\eta_{0}$ is the induced labeling of $B_{\mathrm{sm}}$. By Riemann's existence theorem [27, Section 2.2], $\beta_{\gamma}^{\circ} \otimes \mathbb{C}: \widetilde{\mathcal{M}}_{G}^{\gamma, \circ} \otimes \mathbb{C} \rightarrow \widetilde{\mathcal{M}}_{0, r}^{\circ} \otimes \mathbb{C}$ is an isomorphism. The statement follows since $\widetilde{\mathcal{M}}_{0, r}^{\circ} \otimes \mathbb{C}$ is irreducible.

If two class vectors $\gamma$ and $\gamma^{\prime}$ yield the same inertia type, so that $\bar{\gamma}=\bar{\gamma}^{\prime}$, then there is a permutation $\varpi$ of $\{1, \ldots, r\}$ such that $\gamma^{\prime}=\gamma \circ \varpi$. This relabeling of the branch locus yields an isomorphism

$$
\widetilde{\mathcal{M}}_{G}^{\gamma} \stackrel{\sim}{\longrightarrow} \widetilde{\mathcal{M}}_{G}^{\gamma \circ \varpi}
$$

Suppose $\gamma$ and $\gamma^{\prime}$ differ by an automorphism of $G$, so that there exists $\sigma \in \operatorname{Aut}(G)$ such that $\gamma^{\prime}=\sigma \circ \gamma$. This relabeling of the G-action yields an isomorphism

$$
\widetilde{\mathcal{M}}^{\gamma} \stackrel{\sim}{\longrightarrow} \widetilde{\mathcal{M}}_{G}^{\sigma \circ \gamma}
$$

Lemma 2.3. The forgetful functor $\widetilde{\mathcal{M}}_{G}^{\gamma} \rightarrow \overline{\mathcal{M}}_{G}^{\bar{\gamma}}$ is étale and Galois.

Proof. The map is étale since any admissible stable G-curve admits a labeling of $R_{\mathrm{sm}}$ étalelocally on the base. Moreover, the set of labelings of a fixed stable G-curve with rational smooth ramification locus is a torsor under the subgroup of $\operatorname{Sym}(r)$ consisting of those $\varpi$ for which $\gamma=\gamma \circ \varpi$. Therefore, $\widetilde{\mathcal{M}}_{G}^{\gamma} \rightarrow \overline{\mathcal{M}}_{G}^{\gamma}$ is Galois.

\subsection{Degeneration}

For $i=1,2,3$, let $\gamma_{i}$ denote a class vector with length $r_{i}$ and let $g_{i}=g\left(\gamma_{i}\right)$. There is a clutching map

$$
\widetilde{\mathcal{M}}_{g_{1}, r_{1}} \times \widetilde{\mathcal{M}}_{g_{2}, r_{2}} \rightarrow \widetilde{\mathcal{M}}_{g_{1}+g_{2}, r_{1}+r_{2}-2}
$$

which is a closed immersion [18, 3.9]. On $S$-points, this map corresponds to gluing $C_{1}$ and $C_{2}$ together over $S$ by identifying the last section of $C_{1}$ with the first section of $C_{2}$.

There is a forgetful functor $\widetilde{\mathcal{M}}_{G}^{\gamma} \rightarrow \widetilde{\mathcal{M}}_{g(\gamma), r(\gamma)}$ taking the isomorphism class of $\left(C / S, \iota_{0}, \eta\right)$ to the isomorphism class of $\left(C / S, R_{\mathrm{sm}}, \eta\right)$. This functor is finite-to-one since $\operatorname{Aut}_{S}\left(C, \iota_{0}\right)$ is finite [8, 1.11]. The composition

$$
\widetilde{\mathcal{M}}_{G}^{\gamma_{1}} \times \widetilde{\mathcal{M}}_{G}^{\gamma_{2}} \rightarrow \widetilde{\mathcal{M}}_{g_{1}, r_{1}} \times \widetilde{\mathcal{M}}_{g_{2}, r_{2}} \rightarrow \widetilde{\mathcal{M}}_{g_{1}+g_{2}, r_{1}+r_{2}-2}
$$


allows us to glue two labeled $G$-curves $\left(C_{i} / S, \iota_{0, i}, \eta_{i}\right)$ together to obtain a labeled $G$-curve $C / S$ with genus $g_{1}+g_{2}$ and class vector

$$
\gamma=\left(\gamma_{1}(1), \ldots, \gamma_{1}\left(r_{1}-1\right), \gamma_{2}(2), \ldots, \gamma_{2}\left(r_{2}\right)\right)
$$

Moreover, $C / S$ is equivariantly smoothable if and only if the $G$-action is admissible, i.e., if and only if $\gamma_{1}(r)$ and $\gamma_{2}(1)$ are inverses [10, 2.2]. In this situation, we say that $\left(\gamma_{1}, \gamma_{2}\right)$ deforms to $\gamma$ or that $\gamma$ degenerates to $\left(\gamma_{1}, \gamma_{2}\right)$, and write

$$
\widetilde{\mathcal{M}}_{G}^{\gamma_{1}} \times \widetilde{\mathcal{M}}_{G}^{\gamma_{2}} \longrightarrow \widetilde{\mathcal{M}}_{G}^{\gamma}
$$

The clutching maps admit a generalization to maps

$$
\widetilde{\mathcal{M}}_{g_{1}, r_{1}} \times \widetilde{\mathcal{M}}_{g_{2}, r_{2}} \times \widetilde{\mathcal{M}}_{g_{3}, r_{3}} \rightarrow \widetilde{\mathcal{M}}_{g_{1}+g_{2}+g_{3}, r_{1}+r_{2}+r_{3}-4}
$$

Assume that $\left(\gamma_{1}, \gamma_{2}\right)$ deforms to $\gamma_{L}$, that $\left(\gamma_{2}, \gamma_{3}\right)$ deforms to $\gamma_{R}$, and that both $\left(\gamma_{L}, \gamma_{3}\right)$ and $\left(\gamma_{1}, \gamma_{R}\right)$ deform to $\gamma$. Then we have a commutative diagram of maps:

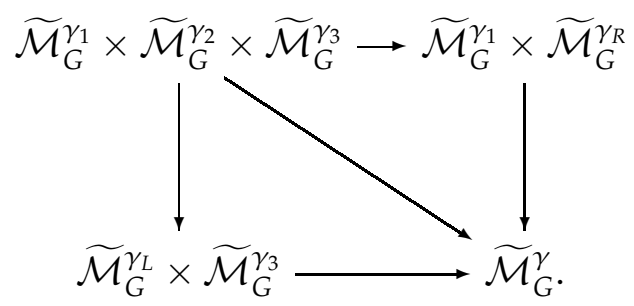

In the situation above, suppose $g_{1}=g_{3}=1$. The image $\Delta_{1,1}$ of $\widetilde{\mathcal{M}}_{G}^{\gamma_{1}} \times \widetilde{\mathcal{M}}_{G}^{\gamma_{2}} \times \widetilde{\mathcal{M}}_{G}^{\gamma_{3}}$ in $\widetilde{\mathcal{M}}_{G}^{\gamma}$ is part of the boundary of $\widetilde{\mathcal{M}}_{G}^{\gamma}$.

We say that a class vector $\gamma$ degenerates to $\Delta_{1,1}$ if there are $\gamma_{1}, \gamma_{2}$ and $\gamma_{3}$ as in (2.3) so that $g_{1}=g_{3}=1$. Furthermore, we say that an inertia type $\bar{\gamma}$ degenerates to $\Delta_{1,1}$ if there exists some class vector $\gamma^{\prime}$ with inertia type $\bar{\gamma}^{\prime}=\bar{\gamma}$ so that $\gamma^{\prime}$ degenerates to $\Delta_{1,1}$. Using Equations (2.1) and (2.2), if $\bar{\gamma}$ degenerates to $\Delta_{1,1}$, then every component of $\widetilde{\mathcal{M}}_{G}$ lying over $\overline{\mathcal{M}}_{G}^{\bar{\gamma}}$ degenerates to $\Delta_{1,1}$ (although this may require identifying different ramification points of $C_{1}, C_{2}$ and $C_{3}$ in the clutching maps).

\subsection{The case of hyperelliptic curves: $d=2$}

Let $C / S$ be a $\mathbb{Z} / 2$-curve of genus $g$. Then $C / S$ is hyperelliptic, and $\iota_{0}(1)$ is the hyperelliptic involution. Over every geometric point $s$ of $S, C_{s} \rightarrow C_{s} / \iota_{0}(\mathbb{Z} / 2)$ is ramified at $2 g+2$ smooth points.

There is a unique class vector $(1, \ldots, 1)$ for $\mathbb{Z} / 2$ of length $2 g+2$ for each $g \in \mathbb{N}$. By Lemma 2.2, there is a unique component of $\widetilde{\mathcal{M}}_{\mathbb{Z} / 2}$ parametrizing labeled hyperelliptic curves of genus $g$. We denote this component by $\widetilde{\mathcal{H}}_{g}$. Similarly, $\overline{\mathcal{M}}_{\mathbb{Z} / 2}$ has a unique component $\overline{\mathcal{H}}_{g}$ which parametrizes hyperelliptic curves of genus $g$. Let $\mathcal{H}_{g}=\overline{\mathcal{H}}_{g}^{\circ}$ denote the moduli space of smooth hyperelliptic curves of genus $g$.

Lemma 2.4. If $g \geq 3$, then $\widetilde{\mathcal{H}}_{g}$ degenerates to $\Delta_{1,1}$. 
Proof. The class vector for $\widetilde{\mathcal{H}}_{g}$ is $\gamma=(1, \ldots, 1)$ with length $2 g+2$. Let $\gamma_{1}=\gamma_{3}=$ $(1,1,1,1)$ and let $\gamma_{2}=(1, \ldots, 1)$ with length $2 g-2$.

Remark 2.5. When the boundary components $\Delta_{1}$ and $\Delta_{2}$ are distinct, the inductive argument in Theorem 3.4 can be revised to rely on monodromy groups only up to isomorphism. Unfortunately, when $g=3$ one has $\Delta_{1}=\Delta_{2}$, so that we must calculate monodromy groups exactly.

\subsection{The case of trielliptic curves: $d=3$}

A trielliptic curve is a $\mathbb{Z} / 3$-curve $\left(C / S, \iota_{0}\right)$ so that $C / \iota_{0}(\mathbb{Z} / 3)$ has genus zero. The curve $C / S$ is sometimes called a cyclic trigonal curve. Over every geometric point $s$ of $S$, the $\mathbb{Z} / 3$-cover $C_{s} \rightarrow C_{s} / \iota_{0}(\mathbb{Z} / 3)$ is ramified at $g+2$ smooth points.

\subsubsection{Components of the trielliptic locus}

The moduli space of trielliptic curves $\left(C / S, \iota_{0}\right)$ with $C$ of genus $g$ is not connected even for fixed $g$. This reflects the different possibilities for the inertia type, or equivalently for the signature, which we now describe.

By Lemma 2.2, the components of $\widetilde{\mathcal{M}}_{\mathbb{Z} / 3}$ parametrizing labeled trielliptic curves of genus $g$ are in bijection with the maps $\gamma:\{1, \ldots, g+2\} \rightarrow(\mathbb{Z} / 3)^{\times}$such that $\sum \gamma(i)=$ $0 \in \mathbb{Z} / 3$. We denote these components by $\widetilde{\mathcal{T}}^{\gamma}$, and their projections to $\overline{\mathcal{M}}_{\mathbb{Z} / 3}$ by $\overline{\mathcal{T}}^{\bar{\gamma}}$. Let $\mathcal{T}^{\bar{\gamma}}=\overline{\mathcal{T}}^{\bar{\gamma} \circ}$ denote the moduli space of smooth trielliptic curves with inertia type $\bar{\gamma}$.

Lemma 2.6. The set of possible inertia types $\bar{\gamma}$ for a trielliptic curve $\left(C / S, \iota_{0}\right)$ of genus $g$ is in bijection with the set of pairs of integers $\left(d_{1}, d_{2}\right)$ so that $d_{1}, d_{2} \geq 0, d_{1}+d_{2}=g+2$, and $d_{1}+2 d_{2} \equiv 0 \bmod 3$.

Proof. Let $d_{1}=\bar{\gamma}(1)$ and $d_{2}=\bar{\gamma}(2)$. The claim follows from earlier remarks.

Remark 2.7. The result [12, 5.5] applies to trielliptic curves only when $d_{1} d_{2}=0$. These inertia types occur for curves of genus $3 g_{1}+1$ having an equation of the form $y^{3}=f(x)$ for some separable polynomial $f(x)$ of degree $3 g_{1}+2$.

Let $S$ be an irreducible scheme over $\operatorname{Spec} \mathbb{Z}\left[1 / 3, \zeta_{3}\right]$. Then $\mathcal{O}_{S} \otimes \mathbb{Z}\left[1 / 3, \zeta_{3}\right] \cong \mathcal{O}_{S} \oplus \mathcal{O}_{S}$. We choose the isomorphism so that the first component has the given structure of $\mathcal{O}_{S}$ as a $\mathbb{Z}\left[1 / 3, \zeta_{3}\right]$-module.

Consider a trielliptic curve $\left(\psi: C \rightarrow S, \iota_{0}\right)$. The sheaf of relative one-forms $\psi_{*} \Omega_{C / S}^{1}$ is a locally free $\mathbb{Z}\left[1 / 3, \zeta_{3}\right] \otimes \mathcal{O}_{S}$-module of some rank $(r, s)$, where $r+s=g$. We call $(r, s)$ the signature of $\left(C / S, \iota_{0}\right)$. The signature is locally constant on $S$, so we may calculate it at any geometric point of $S$. If $\left(C / k, \iota_{0}\right)$ is a trielliptic curve, then $H^{0}\left(C, \Omega_{C}^{1}\right)$ decomposes as a direct sum $W_{1} \oplus W_{2}$ where $\omega \in W_{j}$ if $\zeta_{3} \circ \omega=\zeta_{3}^{j} \omega$. The signature of $\left(C / k, \iota_{0}\right)$ is $(r, s)=\left(\operatorname{dim}\left(W_{1}\right), \operatorname{dim}\left(W_{2}\right)\right)$.

Lemma 2.8. The signature and inertia type of a trielliptic curve of genus $g$ are related as follows: $\bar{\gamma}(1)=2 r-s+1$ and $\bar{\gamma}(2)=2 s-r+1$. There exists a trielliptic curve $\left(C / k, \iota_{0}\right)$ of genus $g$ with signature $(r, s)$ if and only if $r, s \in \mathbb{Z}, r+s=g$, and $(g-1) / 3 \leq r, s \leq(2 g+1) / 3$. 
Proof. Let $\left(C / k, \iota_{0}\right)$ be a trielliptic curve with inertia type $\bar{\gamma}$. For simplicity, let $d_{1}=\bar{\gamma}(1)$ and let $d_{2}=\bar{\gamma}(2)$ and let $N=\left(d_{1}+2 d_{2}\right) / 3$. There is an equation for $C$ of the form

$$
y^{3}=\prod_{i=1}^{d_{1}}\left(x-a_{i}\right) \prod_{j=1}^{d_{2}}\left(x-b_{j}\right)^{2} .
$$

Consider the differential $\omega=\prod_{i=1}^{d_{1}}\left(x-a_{i}\right)^{n_{i}} \prod_{j=1}^{d_{2}}\left(x-b_{j}\right)^{n_{j}^{\prime}} d x / y^{m}$ with $n_{i}, n_{j}^{\prime} \geq 0$ and $m \geq 1$. By [19. Thm. 3], $\omega$ is holomorphic if and only if $3 n_{i} \geq m-2,3 n_{j}^{\prime} \geq 2 m-2$, and $m N \geq \sum_{i=1}^{d_{1}} n_{i}+\sum_{j=1}^{d_{2}} n_{j}^{\prime}+2$. Let $g(x)=\prod_{j=1}^{d_{2}}\left(x-b_{j}\right)$. Thus we have the following set of linearly independent holomorphic differentials:

$$
\left\{d x / y, \ldots,\left(x-a_{1}\right)^{N-2} d x / y, g(x) d x / y^{2}, \ldots,\left(x-a_{1}\right)^{2 N-2-d_{2}} g(x) d x / y^{2}\right\} .
$$

This set is a basis of $H^{0}\left(C, \Omega_{C}^{1}\right)$ since its cardinality is $3 N-d_{2}-2=g$. Also, for any $h(x) \in k[x], \zeta_{3} \circ h(x) d x / y^{m}=\zeta_{3}^{-m} h(x) d x / y^{m}$. It follows that the signature of $\left(C / k, \iota_{0}\right)$ is $(r, s)=(g-N+1, N-1)$.

It follows that $d_{1}=2 r-s+1$ and $d_{2}=2 s-r+1$. Now $r, s \in \mathbb{Z}$ if and only if $d_{1}+2 d_{2} \equiv 0 \bmod 3$. Also, $d_{1}+d_{2}=g+2$ if and only if $r+s=g$. The conditions $d_{1}, d_{2} \geq 0$ and $(g-1) / 3 \leq r, s \leq(2 g+1) / 3$ are equivalent. The second claim then follows from Lemma 2.6

Definition 2.9. Let $g \in \mathbb{N}$. A trielliptic signature for $g$ is a pair $(r, s)$ with $r, s \in \mathbb{Z}, r+s=g$, and $(g-1) / 3 \leq r, s \leq(2 g+1) / 3$.

As in (2.2), if $\left(C / S, \iota_{0}\right)$ is a trielliptic curve then so is $\left(C / S, \iota_{0}^{\prime}\right)$ where $\iota_{0}^{\prime}(1)=\iota_{0}(2)$. Replacing $\iota_{0}$ with $\iota_{0}^{\prime}$ exchanges the values of $d_{1}$ and $d_{2}$ and the values of $r$ and $s$.

\subsubsection{Degeneration of the trielliptic locus}

We show that every component $\widetilde{\mathcal{T}} \gamma$ with $g(\gamma) \geq 4$ degenerates to $\Delta_{1,1}$.

Recall that there is a unique elliptic curve $E$ which admits a $\mathbb{Z} / 3$-action $\iota_{0}$. The class vector of $\left(E, \iota_{0}\right)$ is either $(1,1,1)$ or $(2,2,2)$.

Proposition 2.10. If $g(\gamma) \geq 4$, then $\tilde{\mathcal{T}}^{\gamma}$ degenerates to $\Delta_{1,1}$.

Proof. Let $g=g(\gamma) \geq 4$ and let $\gamma:\{1, \ldots, g+2\} \rightarrow(\mathbb{Z} / 3)^{\times}$be a class vector for $G=\mathbb{Z} / 3$. It suffices to show that there exist class vectors $\gamma_{i}$ for $i=1,2,3$ with $g\left(\gamma_{1}\right)=$ $g\left(\gamma_{3}\right)=1$ as in (2.3). In particular, we require that $\gamma_{1}$ and $\gamma_{3}$ are either $(1,1,1)$ or $(2,2,2)$.

By Equations (2.1) and (2.2), we can reorder the values $\gamma(i)$ or replace each $\gamma(i)$ with $-\gamma(i)$. Thus it is sufficient to restrict attention to the inertia type $\bar{\gamma}$, or to the corresponding signature $(r, s)$ by Lemma 2.8 .

Suppose $\gamma_{1}=\gamma_{3}=(1,1,1)$. Then we need $\gamma_{2}:\{1, \ldots, g\} \rightarrow(\mathbb{Z} / 3)^{\times}$with $\gamma_{2}(1)=$ $\gamma_{2}(g)=2$ and $\bar{\gamma}_{2}(1)=\bar{\gamma}(1)-4$ and $\bar{\gamma}_{2}(2)=\bar{\gamma}(2)+2$. In other words, we need $\gamma_{2}$ to have four fewer points with inertia generator 1 and two more points with inertia generator 2 than $\gamma$ does. This can be achieved by decreasing $r$ by 2 . Similarly, the case $\gamma_{1}=\gamma_{3}=(2,2,2)$ can be achieved by decreasing $s$ by 2 . 
Suppose $\gamma_{1}=(1,1,1)$ and $\gamma_{2}=(2,2,2)$. Then we need $\gamma_{2}:\{1, \ldots, g\} \rightarrow(\mathbb{Z} / 3)^{\times}$ with $\gamma_{2}(1)=2$ and $\gamma_{2}(g)=1$ and $\bar{\gamma}_{2}(1)=\bar{\gamma}(1)-1$ and $\bar{\gamma}_{2}(2)=\bar{\gamma}(2)-1$. In other words, we need $\gamma_{2}$ to have one fewer point with inertia generator 1 and one fewer point with inertia generator 2 than $\gamma$ does. This can be done by decreasing both $r$ and $s$ by 1 .

The next table shows that, for each inertia type $\bar{\gamma}$, there is a choice of $\gamma_{1}, \gamma_{2}, \gamma_{3}$ satisfying the numerical constraints.

\begin{tabular}{|l|l|l|l|l|}
\hline $\begin{array}{l}\text { Component } \gamma \\
\text { with signature }\end{array}$ & $\begin{array}{l}\text { degenerates so that } \\
\gamma_{2} \text { has signature }\end{array}$ & and $\gamma_{1}$ is & and $\gamma_{3}$ is & $\begin{array}{l}\text { under this } \\
\text { condition }\end{array}$ \\
\hline \hline$(r, s)$ & $(r-2, s)$ & $(1,1,1)$ & $(1,1,1)$ & $r \geq 2$ \\
\hline$(r, s)$ & $(r, s-2)$ & $(2,2,2)$ & $(2,2,2)$ & $s \geq 2$ \\
\hline$(r, s)$ & $(r-1, s-1)$ & $(1,1,1)$ & $(2,2,2)$ & $r \geq 1, s \geq 1$ \\
\hline
\end{tabular}

Remark 2.11. When $\widetilde{\mathcal{T}}^{\gamma}$ degenerates to both $\Delta_{1}$ and $\Delta_{2}$, the inductive argument in Theorem 3.9 can be revised to rely on monodromy groups only up to isomorphism. Unfortunately, $\tilde{\mathcal{T}}^{\gamma}$ does not degenerate to $\Delta_{2}$ when $\bar{\gamma}(1) \bar{\gamma}(2)=0$. For $g(\gamma) \geq 4$, all other components $\widetilde{\mathcal{T}}^{\gamma}$ degenerate to $\Delta_{2}$.

\section{Monodromy groups}

\subsection{Definition of monodromy}

Let $(X / S, \phi)$ be a principally polarized abelian scheme of relative dimension $g$ over an irreducible base. If $\ell$ is a rational prime invertible on $S$, then the $\ell$-torsion $X[\ell]$ of $\ell$ is an étale cover of $S$ with geometric fiber isomorphic to $(\mathbb{Z} / \ell)^{2 g}$. Let $s$ be a geometric point of $S$. The fundamental group $\pi_{1}(S, s)$ acts linearly on the $\ell$-torsion of $X$. This yields a representation

$$
\rho_{X \rightarrow S, s, \ell}: \pi_{1}(S, s) \rightarrow \operatorname{Aut}\left(X[\ell]_{s}\right) \cong \mathrm{GL}_{2 g}(\mathbb{Z} / \ell) .
$$

The cover $X[\ell] \rightarrow S$ both determines and is determined by the representation $\rho_{X \rightarrow S, s, \ell}$. The image of $\rho_{X \rightarrow S, s, \ell}$ is the mod- $\ell$ monodromy of $X \rightarrow S$ and we denote it by $\mathrm{M}_{\ell}(X \rightarrow S, s)$, or by $\mathrm{M}_{\ell}(S, s)$ if the choice of abelian scheme is clear. The isomorphism class of the $\mathrm{M}_{\ell}(S, s)$ is independent of the choice of base point $s$, and we denote it by $\mathrm{M}_{\ell}(S)$.

Let $X^{\vee}$ be the dual abelian scheme. There is a canonical pairing $X[\ell] \times X^{\vee}[\ell] \rightarrow$ $\boldsymbol{\mu}_{\ell, S}$, where $\boldsymbol{\mu}_{\ell, S}:=\boldsymbol{\mu}_{\ell} \times S$ is the group scheme of $\ell^{\text {th }}$ roots of unity. The polarization $\phi$ induces an isomorphism $X \rightarrow X^{\vee}$, and thus a skew-symmetric pairing $X[\ell] \times X[\ell] \rightarrow$ $\boldsymbol{\mu}_{\ell, s}$. Because the polarization is defined globally, the image of monodromy $\mathrm{M}_{\ell}(X \rightarrow S, s)$ is contained in the group of symplectic similitudes of $\left(X[\ell]_{S},\langle\cdot, \cdot\rangle_{\phi}\right)$, which is isomorphic to $\mathrm{GSp}_{2 g}(\mathbb{Z} / \ell)$. Moreover, if a primitive $\ell^{\text {th }}$ root of unity exists globally on $S$, then $\pi_{1}(S, s)$ acts trivially on $\boldsymbol{\mu}_{\ell, S}$ and $\mathrm{M}_{\ell}(X \rightarrow S, s) \subseteq \operatorname{Sp}\left(X[\ell]_{S},\langle\cdot, \cdot\rangle_{\phi}\right) \cong \operatorname{Sp}_{2 g}(\mathbb{Z} / \ell)$.

Similarly, the cover $X\left[\ell^{n}\right] \rightarrow S$ defines a monodromy representation with values in $\operatorname{Aut}\left(X\left[\ell^{n}\right]_{s}\right) \cong \mathrm{GL}_{2 g}\left(\mathbb{Z} / \ell^{n}\right)$. Taking the inverse limit over all $n$, we obtain a continuous 
representation on the Tate module of $X$,

$$
\rho_{X \rightarrow S, \mathbb{Z}_{\ell, s}}: \pi_{1}(S, s) \longrightarrow \lim _{\overleftarrow{n}} \operatorname{Aut}\left(X\left[\ell^{n}\right]_{s}\right) \cong \mathrm{GL}_{2 g}\left(\mathbb{Z}_{\ell}\right)
$$

We denote the image of this representation by $\mathrm{M}_{\mathbb{Z}_{\ell}}(X \rightarrow S, s)$, and its isomorphism class by $\mathrm{M}_{\mathbb{Z}_{\ell}}(X \rightarrow S)$ or $\mathrm{M}_{\mathbb{Z}_{\ell}}(S)$. Again, there is an inclusion $\mathrm{M}_{\mathbb{Z}_{\ell}}(X \rightarrow S) \subseteq \mathrm{GSp}_{2 g}\left(\mathbb{Z}_{\ell}\right)$. If $F$ is a field, let $F_{\ell^{\infty}}=F\left(\boldsymbol{\mu}_{\ell^{\infty}}(\bar{F})\right)$. If $S$ is an $F$-scheme, then $\mathrm{M}_{\mathbb{Z}_{\ell}}(X \rightarrow S, s) / \mathrm{M}_{\mathbb{Z}_{\ell}}(X \otimes \bar{F} \rightarrow$ $S \otimes \bar{F}, s) \cong \operatorname{Gal}\left(F_{\ell^{\infty}} / F\right)$. Finally, let $\mathrm{M}_{\mathbb{Q}_{\ell}}(X \rightarrow S, s)$ be the Zariski closure of $\mathrm{M}_{\mathbb{Z}_{\ell}}(X \rightarrow S, s)$ in $\mathrm{GL}_{2 g}\left(\mathbb{Q}_{\ell}\right)$.

Now suppose that $\psi: C \rightarrow S$ is a relative proper semi-stable curve. Let $\operatorname{Pic}^{0}(C):=$ $\mathrm{Pic}_{C / S}^{0}$ be the neutral component of the relative Picard functor of $C$ over $S$. Since $C / S$ is semi-stable, $\operatorname{Pic}^{0}(C)$ is a semiabelian scheme [4, 9.4.1]. Suppose that there is at least one geometric point $s$ such that the fiber $\operatorname{Pic}^{0}\left(C_{s}\right)$ is an abelian variety. (This is true if some $C_{S}$ is a tree of smooth curves.) Then there is a nonempty open subscheme $S^{*}$ of $S$ such that $\operatorname{Pic}^{0}\left(\left.C\right|_{S^{*}}\right)$ is an abelian scheme over $S^{*}$. We define the mod- $\ell$ and $\mathbb{Z}_{\ell}$ monodromy representations of $C$ to be those of $\operatorname{Pic}^{0}\left(\left.C\right|_{S^{*}}\right) \rightarrow S^{*}$. (Alternatively, these may be constructed as the restrictions of $R^{1} \psi_{*} \boldsymbol{\mu}_{\ell, S}$ and $R^{1} \psi_{*} \boldsymbol{\mu}_{\ell \infty, S}$ to the largest subscheme of $S$ on which these sheaves are unramified.) Thus, $\mathrm{M}_{\ell}(C \rightarrow S, s)=\mathrm{M}_{\ell}\left(\operatorname{Pic}^{0}\left(\left.C\right|_{S^{*}}\right) \rightarrow S^{*}, s\right)$, and we denote this again by $\mathrm{M}_{\ell}(S, s)$ if the curve is clear and by $\mathrm{M}_{\ell}(S)$ if the base point is suppressed.

The moduli spaces $\overline{\mathcal{M}}_{G}$ and $\widetilde{\mathcal{M}}_{G}$ are Deligne-Mumford stacks, and we employ a similar formalism for étale covers of stacks |24|. Let $\mathcal{S}$ be a connected Deligne-Mumford stack. The category of Galois étale covers of $\mathcal{S}$ is a Galois category in the sense of Grothendieck, and thus there is an étale fundamental group of $\mathcal{S}$. More precisely, let $s \in \mathcal{S}$ be a geometric point. Then there is a group $\pi_{1}(\mathcal{S}, s)$ and an equivalence of categories between finite $\pi_{1}(\mathcal{S}, s)$-sets and finite étale Galois covers of $\mathcal{S}$. If $\mathcal{S}$ has a coarse moduli space $S_{\text {mod, }}$, then $\pi_{1}(\mathcal{S}, s)$ is the extension of $\pi_{1}\left(S_{\text {mod }}, s\right)$ by a group which encodes the extra automorphism structure on the moduli space $S_{\text {mod }}$ [24. 7.11]. If $X \rightarrow \mathcal{S}$ is a family of abelian varieties, we again let $\mathrm{M}_{\ell}(X \rightarrow \mathcal{S}, s)$ be the image of $\pi_{1}(\mathcal{S}, s)$ in $\operatorname{Aut}\left(X[\ell]_{s}\right)$.

Let $\mathcal{C}^{\gamma}$ be the tautological labeled curve over $\widetilde{\mathcal{M}}_{G}^{\gamma}$. By the mod- $\ell$ or $\mathbb{Z}_{\ell}$ monodromy of $\widetilde{\mathcal{M}}_{G}^{\gamma}$ we mean that of $C^{\gamma} \rightarrow \widetilde{\mathcal{M}}_{G}^{\gamma}$.

\subsection{Degeneration and monodromy}

In this section, we deduce information on monodromy groups from the degeneration of moduli spaces to the boundary. We will use this to prove inductively that the mod- $\ell$ monodromy groups of each $\widetilde{\mathcal{H}}_{g}$ and each $\widetilde{\mathcal{T}}^{\gamma}$ are as large as possible.

Lemma 3.1. Let $k$ be an algebraically closed field in which $d \ell$ is invertible. Suppose that the pair $\left(\gamma_{1}, \gamma_{2}\right)$ deforms to $\gamma$, so that there is a clutching map

$$
\kappa: \widetilde{\mathcal{M}}_{G}^{\gamma_{1}} \times \widetilde{\mathcal{M}}_{G}^{\gamma_{2}} \longrightarrow \widetilde{\mathcal{M}}_{G}^{\gamma} .
$$

a. There is a canonical isomorphism of sheaves on $\left(\widetilde{\mathcal{M}}_{G}^{\gamma_{1}} \times \widetilde{\mathcal{M}}_{G}^{\gamma_{2}}\right) \otimes k$,

$$
(\kappa \otimes k)^{*} \operatorname{Pic}^{0}\left(\mathcal{C}^{\gamma}\right)[\ell] \cong \operatorname{Pic}^{0}\left(\mathcal{C}^{\gamma_{1}}\right)[\ell] \times \operatorname{Pic}^{0}\left(\mathcal{C}^{\gamma_{2}}\right)[\ell] .
$$


b. Suppose $s_{i} \in \widetilde{\mathcal{M}}_{G}^{\gamma_{i}}(k)$ for $i=1,2$, and let $s=\kappa\left(s_{1}, s_{2}\right)$. After base change to $k$, there is a commutative diagram:

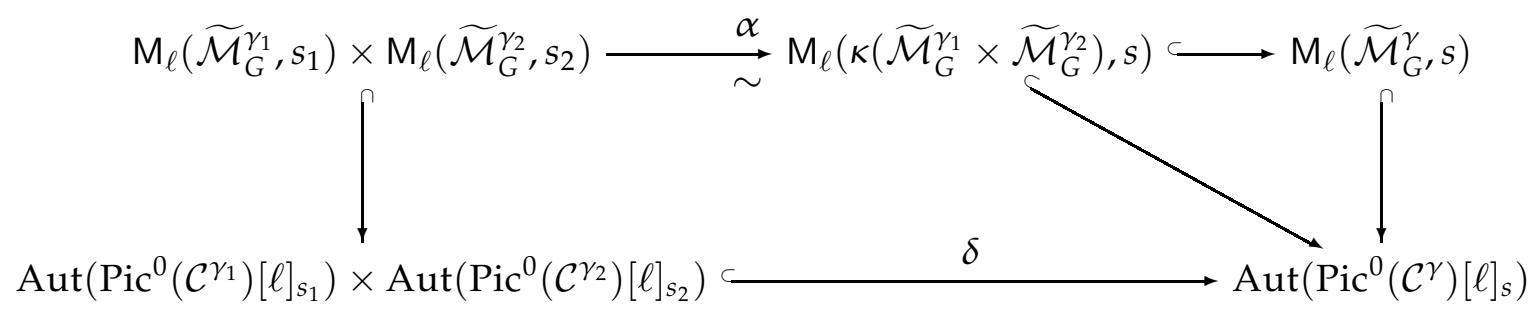

Proof. Let $C \rightarrow S$ be a stable curve. Suppose that $C$ is the union of two (not necessarily irreducible) proper, connected $S$-curves $C_{1}$ and $C_{2}$ which intersect along a unique section. If $\mathcal{L}$ is a line bundle on $C$ of degree zero, then for $i=1,2$ the restriction $\left.\mathcal{L}\right|_{C_{i}}$ is a line bundle of degree zero on $C_{i}$ [4, 9.1.2 and 9.2.13]. Thus, there is a morphism of group functors $(\kappa \otimes k)^{*} \operatorname{Pic}^{0}\left(C^{\gamma}\right) \rightarrow \operatorname{Pic}^{0}\left(C^{\gamma_{1}}\right) \times \operatorname{Pic}^{0}\left(C^{\gamma_{2}}\right)$, which restricts to a morphism on $\ell-$ torsion. This is an isomorphism on stalks [4, 9.2.8], and thus an isomorphism of sheaves.

For part (b), the homomorphism $\delta$ is induced by the isomorphism in Equation (3.1). For $i=1,2$, let $C_{i} \rightarrow S_{i}$ denote the cover $\mathcal{C}^{\gamma_{i}} \otimes k \rightarrow \widetilde{\mathcal{M}}_{G}^{\gamma_{i}} \otimes k$. To define $\alpha$, consider the kernel of the representation $\rho_{C_{i} \rightarrow S_{i}, s_{i}, \ell}$. Since it is an open normal subgroup of the fundamental group, this kernel defines an irreducible Galois étale cover $Y_{i} \rightarrow S_{i}$. Let $H_{i}$ be its Galois group. Then $Y_{1} \times{ }_{k} Y_{2}$ is an irreducible $H_{1} \times H_{2}$ cover of $S_{1} \times S_{2}$. Using this and part (a), the cover $\left.\operatorname{Pic}^{0}\left(\mathcal{C}^{\gamma}\right)[\ell]\right|_{\kappa\left(S_{1} \times S_{2}\right)}$ is trivialized by a Galois extension with group $H_{1} \times H_{2}$. Therefore, $\alpha$ is an isomorphism.

Lemma 3.2. Let $\mathbb{F}$ be a finite field of odd characteristic, and let $V / \mathbb{F}$ be a g-dimensional vector space. Suppose that

$$
V=V_{1} \oplus V_{2} \oplus V_{3}
$$

and that $g_{i}:=\operatorname{dim} V_{i}$ is positive for $i=1,2,3$. Let $H$ be a subgroup of $\operatorname{GL}(V)$.

a. If $H \subseteq \mathrm{SL}(V)$ contains both $\mathrm{SL}\left(V_{1} \oplus V_{2}\right)$ and $\mathrm{SL}\left(V_{2} \oplus V_{3}\right)$, then $H=\operatorname{SL}(V)$.

b. Suppose that $V$ is equipped with a nondegenerate pairing $\langle\cdot, \cdot\rangle$ and that for $i \neq j,\left\langle V_{i}, V_{j}\right\rangle=$ (0).

(i) If the pairing is symplectic, and if $H \subseteq \operatorname{Sp}(V)$ contains both $\operatorname{Sp}\left(V_{1} \oplus V_{2}\right)$ and $\operatorname{Sp}\left(V_{2} \oplus V_{3}\right)$, then $H=\operatorname{Sp}(V)$.

(ii) If the pairing is Hermitian, and if $H \subseteq \mathrm{SU}(V)$ contains both $\mathrm{SU}\left(V_{1} \oplus V_{2}\right)$ and $\mathrm{SU}\left(V_{2} \oplus V_{3}\right)$, then $H=\mathrm{SU}(V)$.

Proof. We use (3.2) to identify $\mathrm{GL}\left(V_{i}\right)$ with a subgroup of $\mathrm{GL}(V)$.

Our proof of (a) is Lie-theoretic. Since SL(V) is split, the roots and Weyl group of $\mathrm{SL}(V)$ are the same as those of $\mathrm{SL}(V \otimes \overline{\mathbb{F}})$ [5, 1.18]. Moreover, $\mathrm{SL}(V)$ has a split BN-pair, and we show $H=\mathrm{SL}(V)$ by successively showing that $H$ contains a maximal torus $T$ of $\mathrm{SL}(V)$, the associated Weyl group, and all root subgroups.

Choose coordinates $e_{1}, \ldots, e_{g}$ so that $V_{1}$ is the span of $\left\{e_{1}, \ldots, e_{g_{1}}\right\} ; V_{2}$ is the span of $\left\{e_{g_{1}+1}, \ldots, e_{g_{1}+g_{2}}\right\}$; and $V_{3}$ is the span of $\left\{e_{g_{1}+g_{2}+1}, \ldots, e_{g}\right\}$. 
First, $H$ contains the maximal split torus $T$ of $S L(V)$ consisting of all diagonal matrices $\operatorname{diag}\left(v_{1}, \ldots, v_{g}\right)$ such that $\prod_{j} v_{j}=1$. To see this, choose $j$ such that $e_{j} \in V_{2}$. Given $v=\operatorname{diag}\left(v_{1}, \ldots, v_{g}\right) \in T$, we use the fact that $v_{j}=\left(\prod_{i \neq j} v\right)^{-1}$ to write

$$
v=\operatorname{diag}\left(v_{1}, \ldots, v_{j-1},\left(\prod_{i=1}^{j-1} v_{i}\right)^{-1}, 1, \ldots, 1\right) \cdot \operatorname{diag}\left(1, \ldots, 1,\left(\prod_{i=j+1}^{g} v_{i}\right)^{-1}, v_{j+1}, \ldots, v_{g}\right) .
$$

Second, $H$ contains $N_{\mathrm{SL}(V)}(T)$, the normalizer of $T$ in $\operatorname{SL}(V)$. It suffices to show $N_{H}(T) / T=N_{\mathrm{SL}(V)}(T) / T$. The normalizer $N_{\mathrm{SL}(V)}(T)$ is the set of matrices of determinant one with exactly one nonzero entry in each row and in each column, and the quotient $N_{\mathrm{SL}(V)}(T) / T$ is isomorphic to $\operatorname{Sym}(\{1, \ldots, g\})$. Under this identification,

$$
\begin{aligned}
& N_{\mathrm{SL}\left(V_{1} \oplus V_{2}\right)}\left(T \cap \operatorname{SL}\left(V_{1} \oplus V_{2}\right)\right) /\left(T \cap \operatorname{SL}\left(V_{1} \oplus V_{2}\right)\right)=\operatorname{Sym}\left(\left\{1, \ldots, g_{1}+g_{2}\right\}\right) ; \\
& N_{\mathrm{SL}\left(V_{2} \oplus V_{3}\right)}\left(T \cap \operatorname{SL}\left(V_{2} \oplus V_{3}\right)\right) /\left(T \cap \operatorname{SL}\left(V_{2} \oplus V_{3}\right)\right)=\operatorname{Sym}\left(\left\{g_{1}+1, \ldots, g\right\}\right) .
\end{aligned}
$$

Since $H$ contains $\operatorname{SL}\left(V_{1} \oplus V_{2}\right)$ and $\operatorname{SL}\left(V_{2} \oplus V_{3}\right)$, it contains $N_{\mathrm{SL}(V)}(T)$.

Third, let $\Delta$ be the canonical set of simple roots associated with $\operatorname{End}(V) \cong \operatorname{Mat}_{n}(V)$. For each $i \in\{1, \ldots, g-1\}$ there is a root $\alpha_{i} \in \Delta$. The associated root group is the unipotent group $U_{\alpha_{i}}$; the nontrivial elements of $U_{\alpha_{i}}$ are unipotent matrices whose only nonzero offdiagonal entry is at $(i, i+1)$. Clearly, $H$ contains each $U_{\alpha_{i}}$.

Finally, the Weyl group $N_{\mathrm{SL}(V)}(T) / T$, acting on the set of simple roots $\Delta$, generates the set of all roots $\Phi$. Since $H$ contains $N_{\mathrm{SL}(V)}(T)$, it therefore contains all root groups $U_{\alpha}$ where $\alpha \in \Phi$. By the Bruhat decomposition, any element of $\operatorname{SL}(V)$ is a product of a diagonal matrix and members of the various root groups. Therefore, $H=\mathrm{SL}(V)$.

For (b) part (i), after relabeling if necessary, we may assume that $V_{1} \oplus V_{2} \neq V_{3}$. Moreover, the hypothesis ensures that $H$ contains $\operatorname{Sp}\left(V_{3}\right)$, and thus it contains $\operatorname{Sp}\left(V_{1} \oplus V_{2}\right) \oplus$ $\mathrm{Sp}\left(V_{3}\right)$. It is known that $\mathrm{Sp}\left(V_{1} \oplus V_{2}\right) \oplus \mathrm{Sp}\left(V_{3}\right)$ is a maximal subgroup of $\mathrm{Sp}(V)$ [17, Thm. 3.2]. Since $\operatorname{Sp}\left(V_{2} \oplus V_{3}\right) \not \subset \mathrm{Sp}\left(V_{1} \oplus V_{2}\right) \oplus \mathrm{Sp}\left(V_{3}\right)$, the subgroup $H$ must equal $\operatorname{Sp}(V)$.

The same argument proves (b) part (ii); as group-theoretic input, we use the fact [17. p.373] that $\mathrm{SU}\left(V_{1} \oplus V_{2}\right) \oplus \mathrm{SU}\left(V_{3}\right)$ is a maximal subgroup of $\mathrm{SU}(V)$.

\subsection{Monodromy of hyperelliptic curves}

We show that the mod- $\ell$ monodromy of the tautological family of hyperelliptic curves of genus $g$ is the full symplectic group $\operatorname{Sp}_{2 g}(\mathbb{Z} / \ell$ ). Recall (Section 2.4) that for $g \in \mathbb{N}$, the moduli space $\widetilde{\mathcal{H}}_{g}$ of labeled hyperelliptic curves of genus $g$ is irreducible. We will use Lemma 3.3 to relate $\mathrm{M}_{\ell}\left(\widetilde{\mathcal{H}}_{g}\right)$ to $\mathrm{M}_{\ell}\left(\overline{\mathcal{H}}_{g}\right)$.

Lemma 3.3. Let $\ell$ be an odd prime. Let $S$ be an irreducible scheme with a primitive $2 \ell^{\text {th }}$ root of unity, and let $(X \rightarrow S, \phi)$ be a principally polarized abelian scheme. Let $Y \rightarrow S$ be a Galois étale cover. Suppose that $\mathrm{M}_{\ell}(X \rightarrow S) \cong \operatorname{Sp}_{2 g}(\mathbb{Z} / \ell)$ and that the groups $\operatorname{Gal}(Y / S)$ and $\operatorname{Sp}_{2 g}(\mathbb{Z} / \ell)$ have no common nontrivial quotients. Then $\mathrm{M}_{\ell}\left(X \times{ }_{S} Y \rightarrow Y\right) \cong \operatorname{Sp}_{2 g}(\mathbb{Z} / \ell)$.

Proof. We equip $(\mathbb{Z} / \ell)^{2 g}$ with the standard symplectic pairing $\langle\cdot, \cdot\rangle_{\text {std }}$. Let

$$
I_{\ell}:=\operatorname{Isom}\left(\left(X[\ell],\langle\cdot, \cdot\rangle_{\phi}\right),\left((\mathbb{Z} / \ell)^{2 g},\langle\cdot, \cdot\rangle_{\text {std }}\right)\right) .
$$


The hypothesis on $S$ implies that $I_{\ell} \rightarrow S$ is an étale Galois cover with group $\operatorname{Sp}_{2 g}(\mathbb{Z} / \ell)$. The hypothesis on $\mathrm{M}_{\ell}(X \rightarrow S)$ implies that $I_{\ell}$ is irreducible.

To prove the lemma, we must show that $I_{\ell} \times{ }_{S} Y$ is irreducible. Equivalently, we must show that $I_{\ell} \rightarrow S$ and $Y \rightarrow S$ are disjoint. Now, $I_{\ell} \times S Y \rightarrow S$ is a (possibly reducible) étale Galois cover with group $\operatorname{Sp}_{2 g}(\mathbb{Z} / \ell) \times \operatorname{Gal}(Y / S)$. If $Z \rightarrow S$ is any common quotient of $I_{\ell} \rightarrow S$ and $Y \rightarrow S$, then so is each conjugate $Z^{\tau} \rightarrow S$ for $\tau \in \operatorname{Gal}\left(I_{\ell} \times{ }_{S} Y / S\right)$. Therefore, the compositum $\widetilde{Z}$ of all such conjugates $Z^{\tau}$ is also a common quotient of $I_{\ell} \rightarrow S$ and $Y \rightarrow S$. It thus suffices to show that there is no nontrivial Galois cover $\widetilde{Z} \rightarrow S$ which is a quotient of $I_{\ell} \rightarrow S$ and $Y \rightarrow S$. This last claim is guaranteed by the group-theoretic hypothesis.

We compute the mod- $\ell$ monodromy of the moduli space of hyperelliptic curves.

Theorem 3.4. Let $\ell$ be an odd prime, and let $k$ be an algebraically closed field in which $2 \ell$ is invertible. For $g \geq 1, \mathrm{M}_{\ell}\left(\widetilde{\mathcal{H}}_{g} \otimes k\right) \cong \mathrm{M}_{\ell}\left(\mathcal{H}_{g} \otimes k\right) \simeq \mathrm{Sp}_{2 g}(\mathbb{Z} / \ell)$.

Proof. Since $\mathcal{H}_{g} \otimes k$ is open and dense in $\overline{\mathcal{H}}_{g} \otimes k$, which is dominated by $\widetilde{\mathcal{H}}_{g} \otimes k$, it suffices to show that $\mathrm{M}_{\ell}\left(\widetilde{\mathcal{H}}_{g} \otimes k\right) \cong \operatorname{Sp}_{2 g}(\mathbb{Z} / \ell)$.

Our proof is by induction on $g$. If $g=1$ or $g=2$, then every curve of genus $g$ is hyperelliptic, so that $\overline{\mathcal{M}}_{g} \otimes k$ and $\overline{\mathcal{H}}_{g} \otimes k$ coincide. Therefore, $\mathrm{M}_{\ell}\left(\overline{\mathcal{H}}_{g} \otimes k\right) \cong \mathrm{M}_{\ell}\left(\overline{\mathcal{M}}_{g} \otimes k\right)$. By [8, 5.15-5.16], $\mathrm{M}_{\ell}\left(\overline{\mathcal{M}}_{g} \otimes k\right) \cong \mathrm{Sp}_{2 g}(\mathbb{Z} / \ell)$.

We conclude the base case $g=1$ and $g=2$ by applying Lemma 3.3 to the cover $\widetilde{\mathcal{H}}_{g} \rightarrow \overline{\mathcal{H}}_{g}$. This cover is étale and Galois, with Galois group $\operatorname{Sym}(2 g+2)$. This group and $\mathrm{Sp}_{2 g}(\mathbb{Z} / \ell)$ have no common nontrivial quotient. (To see this, recall that if $\ell$ is odd then the projective symplectic group $\operatorname{PSp}_{2 g}(\mathbb{Z} / \ell)$ is simple, except that $\operatorname{PSp}_{2}(\mathbb{Z} / 3) \cong A_{4}$. Neither $\operatorname{PSp}_{2 g}(\mathbb{Z} / \ell)$ nor any quotient of $A_{4}$ is a nontrivial quotient of $\operatorname{Sym}(4)$ or $\operatorname{Sym}(6)$.) By Lemma3.3. $\mathrm{M}_{\ell}\left(\widetilde{\mathcal{H}}_{g} \otimes k\right) \cong \mathrm{Sp}_{2 g}(\mathbb{Z} / \ell)$.

We now assume that $g \geq 3$ and that $\mathrm{M}_{\ell}\left(\widetilde{\mathcal{H}}_{g^{\prime}} \otimes k\right) \cong \mathrm{Sp}_{2 g^{\prime}}(\mathbb{Z} / \ell)$ for $1 \leq g^{\prime}<g$. By Lemma 2.4. $\widetilde{\mathcal{H}}_{g} \otimes k$ degenerates to $\Delta_{1,1}$, so that there is a diagram (2.3):

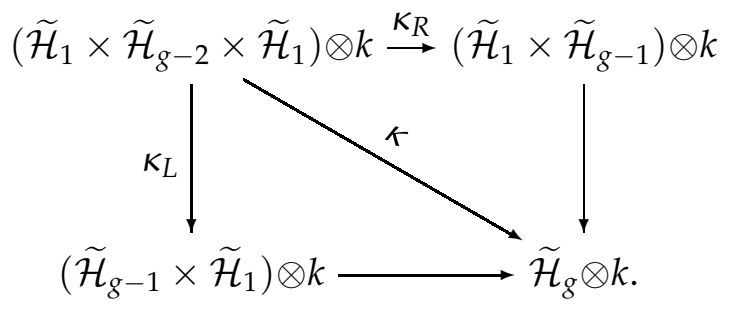

Fix a base point $\left(s_{1}, s_{2}, s_{3}\right) \in\left(\widetilde{\mathcal{H}}_{1} \times \widetilde{\mathcal{H}}_{g-2} \times \widetilde{\mathcal{H}}_{1}\right)(k)$, and let $s=\kappa\left(s_{1}, s_{2}, s_{3}\right)$. For $h \in \mathbb{N}$, let $C^{h} \rightarrow \widetilde{\mathcal{H}}_{h}$ be the tautological labeled $\mathbb{Z} / 2$-curve of genus $h$. Let $V=\operatorname{Pic}^{0}\left(\mathcal{C}^{g}\right)[\ell]_{s}$, and for $i=1,2,3$ let $V_{i}=\operatorname{Pic}^{0}\left(\mathcal{C}^{g_{i}}\right)[\ell]_{s_{i}}$. Each of these is a $\mathbb{Z} / \ell$-vector space equipped with a symplectic form. By Lemma 3.1(a), there is an isomorphism of symplectic $\mathbb{Z} / \ell$-vector spaces $V \cong V_{1} \oplus V_{2} \oplus V_{3}$. 
Let $\widetilde{\mathcal{B}}_{R}$ (resp. $\widetilde{\mathcal{B}}_{L}$ ) be the image of $\kappa_{R}$ (resp. $\kappa_{L}$ ). Using the decomposition in Equation (3.1), we have inclusions:

$$
\begin{aligned}
& \mathrm{M}_{\ell}\left(\widetilde{\mathcal{B}}_{R} \otimes k\right) \subseteq \operatorname{Sp}\left(V_{1}\right) \oplus \operatorname{Sp}\left(V_{2} \oplus V_{3}\right) \\
& \mathrm{M}_{\ell}\left(\widetilde{\mathcal{B}}_{L} \otimes k\right) \subseteq \operatorname{Sp}\left(V_{1} \oplus V_{2}\right) \oplus \operatorname{Sp}\left(V_{3}\right) .
\end{aligned}
$$

By the induction hypothesis, the inclusions in (3.3) are equalities. By Lemma 3.1(b), $\mathrm{M}_{\ell}\left(\widetilde{\mathcal{H}}_{g} \otimes k\right)$ contains $\mathrm{Sp}\left(V_{1}\right) \oplus \mathrm{Sp}\left(V_{2} \oplus V_{3}\right)$ and $\operatorname{Sp}\left(V_{1} \oplus V_{2}\right) \oplus \operatorname{Sp}\left(V_{3}\right)$. By Lemma 3.2b(i), $\mathrm{M}_{\ell}\left(\widetilde{\mathcal{H}}_{g}\right) \cong \mathrm{Sp}_{2 g}(\mathbb{Z} / \ell)$.

Corollary 3.5. Let $\ell$ be an odd prime, and let $k$ be an algebraically closed field in which $2 \ell$ is invertible. For $g \geq 1, \mathrm{M}_{\mathbb{Z}_{\ell}}\left(\widetilde{\mathcal{H}}_{g} \otimes k\right) \cong \mathrm{M}_{\mathbb{Z}_{\ell}}\left(\mathcal{H}_{g} \otimes k\right) \cong \mathrm{Sp}_{2 g}\left(\mathbb{Z}_{\ell}\right)$.

Proof. By Theorem 3.4, $\mathrm{M}_{\ell}\left(\widetilde{\mathcal{H}}_{g} \otimes k\right) \cong \mathrm{Sp}_{2 g}(\mathbb{Z} / \ell)$. By construction, there is a surjection $\mathrm{M}_{\mathbb{Z}_{\ell}}\left(\widetilde{\mathcal{H}}_{g} \otimes k\right) \rightarrow \mathrm{M}_{\ell}\left(\tilde{\mathcal{H}}_{g} \otimes k\right)$. Since the composition $\mathrm{M}_{\mathbb{Z}_{\ell}}\left(\widetilde{\mathcal{H}}_{g} \otimes k\right) \hookrightarrow \mathrm{Sp}_{2 g}\left(\mathbb{Z}_{\ell}\right) \rightarrow \operatorname{Sp}_{2 g}(\mathbb{Z} / \ell)$ is surjective, a standard group theory argument (e.g., [26, 1.3]) shows that $\mathrm{M}_{\mathbb{Z}_{\ell}}\left(\widetilde{\mathcal{H}}_{g} \otimes k\right) \cong$ $\mathrm{Sp}_{2 g}\left(\mathbb{Z}_{\ell}\right)$. As in the proof of Theorem 3.4 $\mathrm{M}_{\mathbb{Z}_{\ell}}\left(\mathcal{H}_{g} \otimes k\right) \cong \mathrm{Sp}_{2 g}\left(\mathbb{Z}_{\ell}\right)$ as well.

Corollary 3.6. Let $p \neq 2$ and let $g \in \mathbb{N}$. Then there exists a smooth hyperelliptic curve of genus $g$ defined over $\overline{\mathbb{F}}_{p}$ whose Jacobian is absolutely simple.

Proof. Let $\ell$ be an odd prime distinct from $p$. By Corollary 3.5 ,

$$
\operatorname{Sp}_{2 g}\left(\mathbb{Z}_{\ell}\right) \cong \mathrm{M}_{\mathbb{Z}_{\ell}}\left(\mathcal{H}_{g} \otimes \overline{\mathbb{F}}_{p}\right) \subseteq \mathrm{M}_{\mathbb{Z}_{\ell}}\left(\mathcal{H}_{g} \otimes \mathbb{F}_{p}\right) \subseteq \operatorname{GSp}_{2 g}\left(\mathbb{Z}_{\ell}\right)
$$

Moreover, $\mathrm{M}_{\mathbb{Z}_{\ell}}\left(\mathcal{H}_{g} \otimes \mathbb{F}_{p}\right) / \mathrm{M}_{\mathbb{Z}_{\ell}}\left(\mathcal{H}_{g} \otimes \overline{\mathbb{F}}_{p}\right) \cong \operatorname{Gal}\left(\mathbb{F}_{p, \ell \infty} / \mathbb{F}_{p}\right)$, which has finite index in $\operatorname{Aut}\left(\boldsymbol{\mu}_{\ell^{\infty}}\left(\overline{\mathbb{F}}_{p}\right)\right) \cong$ $\mathrm{GSp}_{2 g}\left(\mathbb{Z}_{\ell}\right) / \mathrm{Sp}_{2 g}\left(\mathbb{Z}_{\ell}\right)$. Therefore, $\mathrm{M}_{\mathbb{Z}_{\ell}}\left(\mathcal{H}_{g} \otimes \mathbb{F}_{p}\right)$ is an open subgroup of $\mathrm{GSp}_{2 g}\left(\mathbb{Z}_{\ell}\right)$; by Borel's density theorem [25, Th. 4.10], $\mathrm{M}_{\mathbb{Q}_{\ell}}\left(\mathcal{H}_{g} \otimes \mathbb{F}_{p}\right) \cong \mathrm{GSp}_{2 g}\left(\mathbb{Q}_{\ell}\right)$. The claim now follows from [6, Prop. 4].

\subsection{Monodromy of trielliptic curves}

We now compute the monodromy groups of tautological families $\mathcal{C}^{\gamma} \rightarrow \tilde{\mathcal{T}}^{\gamma}$ of labeled trielliptic curves. The Jacobians of trielliptic curves admit an action by $\mathbb{Z}\left[\zeta_{3}\right]$. This places a constraint on $\mathrm{M}_{\ell}\left(\tilde{\mathcal{T}}^{\gamma}\right)$; in Theorem 3.9. we show that this is the only constraint. We need more notation concerning $\mathbb{Z}\left[\zeta_{3}\right]$-actions and unitary groups to describe the monodromy group precisely.

\subsubsection{Unitary groups}

Let $S$ be an irreducible scheme over Spec $\mathbb{Z}\left[1 / 3, \zeta_{3}\right]$, and let $X \rightarrow S$ be an abelian scheme of relative dimension $g$ equipped with an action $\iota: \mathbb{Z}\left[\zeta_{3}\right] \rightarrow \operatorname{End}_{S}(X)$. Then $\operatorname{Lie}(X)$ is a locally free $\mathbb{Z}\left[\zeta_{3}\right] \otimes \mathcal{O}_{S}$-module of some rank $(r, s)$, where $r+s=g$. We call $(r, s)$ the signature of the action of $\mathbb{Z}\left[\zeta_{3}\right]$ on $X$. If $\left(C / S, \iota_{0}\right)$ is a trielliptic curve, then $\operatorname{Lie}\left(\operatorname{Pic}^{0}(C)\right)$ is the $\mathcal{O}_{S}$-linear dual of $\psi_{*} \Omega_{C / S}^{1}$ and the signature of $\operatorname{Pic}^{0}(C)$ is the same as that of $C$. 
Let $V_{(r, s)}$ be a free $\mathbb{Z}\left[\zeta_{3}\right]$-module of rank $g$, equipped with a $\mathbb{Z}\left[\zeta_{3}\right]$-linear pairing $\langle\cdot, \cdot\rangle$ of signature $(r, s)$. Let $\mathrm{GU}_{(r, s)}$ be the $\mathbb{Z}\left[1 / 3, \zeta_{3}\right]$-group scheme of similitudes of the pair $\left(V_{(r, s)},\langle\cdot, \cdot\rangle\right)$, and let $\mathrm{G}_{(r, s)}$ be the restriction of scalars of $\mathrm{GU}_{(r, s)}$ to $\mathbb{Z}[1 / 3]$. Let $\mathrm{SU}_{(r, s)} \subset$ $\mathrm{GU}_{(r, s)}$ be the sub-group scheme of elements of determinant one, and let $\mathrm{SG}_{(r, s)}$ be the restriction of scalars of $S U_{(r, s)}$ to $\mathbb{Z}[1 / 3]$. Concretely, for any $\mathbb{Z}[1 / 3]$-algebra $R$,

$$
\mathrm{SG}_{(r, s)}(R)=\left\{\tau \in \operatorname{Aut}\left(V_{(r, s)} \otimes_{\mathbb{Z}[1 / 3]} R,\langle\cdot, \cdot\rangle\right): \operatorname{det}(\tau)=1\right\} .
$$

In the abstract, we used $\mathrm{SU}_{(r, s)}\left(\mathbb{Z} / \ell \otimes \mathbb{Z}\left[\zeta_{3}\right]\right)$ to denote $\mathrm{SG}_{(r, s)}(\mathbb{Z} / \ell)$. The signature condition implies that $\mathrm{SG}_{(r, s)}(\mathbb{R})$ is isomorphic to the complex special unitary group $\mathrm{SU}(r, s)$.

The behavior of $\mathrm{SG}_{(r, s)}$ at finite primes $\ell \geq 5$ depends on their splitting in $\mathbb{Z}\left[\zeta_{3}\right]$. Specifically, if $\ell$ is inert in $\mathbb{Z}\left[\zeta_{3}\right]$, then $\mathrm{SG}_{(r, s)}(\mathbb{Z} / \ell) \cong \mathrm{SU}_{g}\left(\mathbb{F}_{\ell^{2}}\right)$. Alternatively, suppose that $\ell$ splits in $\mathbb{Z}\left[\zeta_{3}\right]$, and let $\lambda$ and $\bar{\lambda}$ be the two primes of $\mathbb{Z}\left[\zeta_{3}\right]$ lying over $\ell$. The factorization $\ell=\lambda \cdot \bar{\lambda}$ yields a factorization $\mathbb{Z}\left[\zeta_{3}\right] \otimes \mathbb{Z} / \ell \cong \mathbb{Z} / \ell \oplus \mathbb{Z} / \ell$. This induces a decomposition $V_{(r, s)} \otimes \mathbb{Z} / \ell \cong V_{(r, s)}(\lambda) \oplus V_{(r, s)}(\bar{\lambda})$, where $V_{(r, s)}(\lambda)$ and $V_{(r, s)}(\bar{\lambda})$ are $g$-dimensional $\mathbb{Z} / \ell$-vector spaces. Moreover, the inner product $\langle\cdot, \cdot\rangle$ restricts to a perfect pairing between $V_{(r, s)}(\lambda)$ and $V_{(r, s)}(\bar{\lambda})$. Then $\mathrm{SG}_{(r, s)}(\mathbb{Z} / \ell) \subset \operatorname{Aut}\left(V_{(r, s)}(\lambda) \times V_{(r, s)}(\bar{\lambda})\right)$ is the image of $\operatorname{SL}_{g}(\mathbb{Z} / \ell)$ embedded as $\tau \mapsto \tau \times\left({ }^{t} \tau\right)^{-1}$ (where ${ }^{t} \tau$ denotes the transpose of $\tau$ ). In either case, the isomorphism class of $\mathrm{SG}_{(r, s)}(\mathbb{Z} / \ell)$ depends only on $g$ and the splitting of $\ell$ in $\mathbb{Z}\left[\zeta_{3}\right]$, and not on the signature $(r, s)$.

\subsubsection{Calculation of trielliptic monodromy}

Let $\widetilde{\mathcal{T}}^{\gamma}$ be any component of the moduli space of labeled trielliptic curves. The signature of the action of $\mathbb{Z}\left[\zeta_{3}\right]$ on the Lie algebra of the relative Jacobian is locally constant, and we denote it by $\left(r_{\gamma}, s_{\gamma}\right)$ (see Lemma 2.8). The monodromy group $\mathrm{M}_{\ell}\left(\widetilde{\mathcal{T}}^{\gamma} \otimes k\right)$ must preserve both the symplectic pairing and the $\mathbb{Z}\left[\zeta_{3}\right]$-action on the $\ell$-torsion of $\operatorname{Pic}^{0}\left(\mathcal{C}^{\gamma} \otimes k\right)$. This means that there is an inclusion $\mathrm{M}_{\ell}\left(\tilde{\mathcal{T}}^{\gamma} \otimes k\right) \subseteq \mathrm{SG}_{\left(r_{\gamma}, s_{\gamma}\right)}(\mathbb{Z} / \ell)$. We show that this is actually an isomorphism.

As a basis for induction, we compare the moduli space of trielliptic curves of genus three to the Picard modular variety, which is a component of a Shimura variety of PEL type. Let $S h_{(2,1)}$ be the moduli stack parametrizing data $(X / S, l, \phi)$ where $(X / S, \phi)$ is a principally polarized abelian scheme of relative dimension 3 and $\iota: \mathbb{Z}\left[\zeta_{3}\right] \rightarrow \operatorname{End}(X)$ satisfies signature and involution constraints. Specifically, we require that $\operatorname{Lie}(X)$ be a locally free $\mathbb{Z}\left[\zeta_{3}\right] \otimes \mathcal{O}_{S}$-module of signature $(2,1)$ and that $\iota$ take complex conjugation on $\mathbb{Z}\left[\zeta_{3}\right]$ to the Rosati involution on $\operatorname{End}(X)$. Then $\mathcal{S} h_{(2,1)}$ is the Shimura variety associated to the reductive group $\mathrm{G}_{(2,1)}$.

We also consider the moduli stack $\mathcal{S} h_{(2,1), \ell}$ parametrizing data $(X / S, \iota, \phi, \xi)$ where $(X / S, l, \phi)$ is as above and $\xi$ is a principal level $\ell$ structure. More precisely, $\xi$ is a $\mathbb{Z}\left[\zeta_{3}\right]$ linear isomorphism $\xi: X[\ell] \rightarrow\left(V_{(2,1)} \otimes \mathbb{Z} / \ell\right) \otimes \mathcal{O}_{S}$ compatible with the given pairings. The forgetful functor $\mathcal{S} h_{(2,1), \ell} \rightarrow \mathcal{S} h_{(2,1)}$ induces a Galois cover of stacks, with covering group $S G_{(2,1)}(\mathbb{Z} / \ell)$ [13, 1.4]. Note that, as in [11, IV.6.1] but in contrast to [3, 21], we have implicitly chosen an isomorphism $\mu_{\ell} \rightarrow \mathbb{Z} / \ell$. If we had not chosen this isomorphism, the covering group would be $\mathrm{G}_{(2,1)}(\mathbb{Z} / \ell)$; compare [11, IV.6.12]. 
Lemma 3.7. Let $\ell \geq 5$ be prime, and let $S$ be an irreducible scheme with a primitive $3 \ell^{\text {th }}$ root of unity. Let $(X / S, \iota, \phi) \in \mathcal{S} h_{(2,1)}(S)$ be a principally polarized abelian scheme with $\mathbb{Z}\left[\zeta_{3}\right]$-action. Let $Y \rightarrow S$ be an étale Galois cover. Suppose that $\mathrm{M}_{\ell}(X \rightarrow S) \cong \mathrm{SG}_{(2,1)}(\mathbb{Z} / \ell)$ and that the groups $\operatorname{Gal}(Y / S)$ and $\mathrm{SG}_{(2,1)}(\mathbb{Z} / \ell)$ have no common nontrivial quotients. Then $\mathrm{M}_{\ell}\left(X \times{ }_{S} Y \rightarrow\right.$ $Y) \cong \mathrm{SG}_{(2,1)}(\mathbb{Z} / \ell)$.

Proof. The proof is exactly the same as that of Lemma 3.3. except that it involves

$$
I_{\mathbb{Z}\left[\zeta_{3}\right], \ell}:=\operatorname{Isom}_{\mathbb{Z}\left[\zeta_{3}\right]}\left(\left(X[\ell],\langle\cdot, \cdot\rangle_{\phi}\right),\left(V_{2,1} \otimes \mathbb{Z} / \ell,\langle\cdot, \cdot\rangle\right)\right) .
$$

Then $I_{\mathbb{Z}\left[\zeta_{3}\right], \ell} \rightarrow S$ is étale and Galois with group $\mathrm{SU}\left(V_{(2,1)} \otimes \mathbb{Z} / \ell\right) \simeq \mathrm{SG}_{(2,1)}(\mathbb{Z} / \ell)$. The hypothesis on $\mathrm{M}_{\ell}(X \rightarrow S)$ is equivalent to the irreducibility of $I_{\mathbb{Z}\left[\zeta_{3}\right], \ell \text {. }}$

Lemma 3.8. Let $\ell \geq 5$ be prime, and let $k$ be an algebraically closed field in which $3 \ell$ is invertible. Let $\tilde{\mathcal{T}}^{\gamma}$ be any component of the moduli space of trielliptic curves. If $g(\gamma)=3$, then $\mathrm{M}_{\ell}\left(\tilde{\mathcal{T}}^{\gamma} \otimes k\right) \cong \mathrm{SG}_{\left(r_{\gamma}, s_{\gamma}\right)}(\mathbb{Z} / \ell)$.

Proof. We start by computing the monodromy group of the Picard modular variety $\mathcal{S} h_{(2,1)}$. Both $\mathcal{S} h_{(2,1)} \otimes \mathbb{C}$ and $\mathcal{S} h_{(2,1), \ell} \otimes \mathbb{C}$ are arithmetic quotients of the complex ball, thus irreducible [13, 1.4]. Moreover, the existence of smooth arithmetic compactifications [21, Section 3] [3, 1.3.13] and Zariski's connectedness theorem (see [11. IV.5.10]) imply that $\mathcal{S} h_{(2,1)} \otimes k$ and $\mathcal{S} h_{(2,1), \ell} \otimes k$ are also irreducible. The irreducibility of the cover $\mathcal{S} h_{(2,1), \ell} \otimes k \rightarrow$ $\mathcal{S} h_{(2,1)} \otimes k$ implies that the fundamental group of $\mathcal{S} h_{(2,1)} \otimes k$ acts transitively on the set of Hermitian $\mathbb{Z}\left[\zeta_{3}\right] \otimes \mathbb{Z} / \ell$-bases for the $\ell$-torsion of the tautological abelian scheme over $\mathcal{S} h_{(2,1)} \otimes k$. Therefore, $\mathrm{M}_{\ell}\left(\mathcal{S} h_{(2,1)} \otimes k\right) \cong \mathrm{SG}_{(2,1)}(\mathbb{Z} / \ell)$.

We now consider $\widetilde{\mathcal{T}}^{\gamma} \otimes k$. Possibly after relabeling (2.2), by Lemma 2.8 we may assume that $\left(r_{\gamma}, s_{\gamma}\right)=(2,1)$ and that the inertia type $\bar{\gamma}$ is $\{1,1,1,1,2\}$. Moreover, since $\operatorname{dim} \mathcal{S h}(2,1) \otimes k=2$, the Torelli map gives an inclusion of $\mathcal{T}^{\bar{\gamma}}$ onto an open subset of $\mathcal{S} h_{(2,1)}$. Thus $\mathrm{M}_{\ell}\left(\overline{\mathcal{T}}^{\bar{\gamma}} \otimes k\right) \cong \mathrm{M}_{\ell}\left(\mathcal{S} h_{(2,1)} \otimes k\right) \cong \mathrm{SG}_{(2,1)}(\mathbb{Z} / \ell)$.

By Lemma 2.3, $\tilde{\mathcal{T}}^{\gamma} \rightarrow \overline{\mathcal{T}}^{\bar{\gamma}}$ is Galois with group $\operatorname{Sym}(4)$. Since $\ell \geq 5$, the quotient of $\mathrm{SG}_{(2,1)}(\mathbb{Z} / \ell)$ by its center is simple. Thus, $\operatorname{Sym}(4)$ and $\mathrm{SG}_{(2,1)}(\mathbb{Z} / \ell)$ have no common nontrivial quotient, and $\mathrm{M}_{\ell}\left(\widetilde{\mathcal{T}}^{\gamma} \otimes k\right) \cong \mathrm{SG}_{(2,1)}(\mathbb{Z} / \ell)$ by Lemma 3.7

Theorem 3.9. Let $\ell \geq 5$ be prime, and let $k$ be an algebraically closed field in which $3 \ell$ is invertible. Let $\tilde{\mathcal{T}}^{\gamma}$ be any component of the moduli space of labeled trielliptic curves. If $g(\gamma) \geq 3$, then $\mathrm{M}_{\ell}\left(\widetilde{\mathcal{T}}^{\gamma} \otimes k\right) \cong \mathrm{M}_{\ell}\left(\mathcal{T}^{\gamma} \otimes k\right) \cong \mathrm{SG}_{\left(r_{\gamma}, s_{\gamma}\right)}(\mathbb{Z} / \ell)$.

Proof. Since $\mathcal{T}^{\bar{\gamma}} \otimes k$ is open and dense in $\overline{\mathcal{T}}^{\bar{\gamma}} \otimes k$, which is dominated by $\tilde{\mathcal{T}}^{\gamma} \otimes k$, it is sufficient to show $\mathrm{M}_{\ell}\left(\tilde{\mathcal{T}}^{\gamma} \otimes k\right) \cong \mathrm{SG}_{\left(r_{\gamma}, s_{\gamma}\right)}(\mathbb{Z} / \ell)$. We proceed by induction on $g(\gamma)$. The case $g(\gamma)=3$ is supplied by Lemma 3.8

Now let $\widetilde{\mathcal{T}}^{\gamma}$ be any component of $\widetilde{\mathcal{T}}$ with $g=g(\gamma) \geq 4$, and suppose the result is true for all components $\widetilde{\mathcal{T}}^{\gamma^{\prime}}$ with $3 \leq g\left(\gamma^{\prime}\right)<g$. By Proposition 2.10, $\tilde{\mathcal{T}}^{\gamma}$ degenerates to $\Delta_{1,1}$. This means that there are class vectors $\gamma_{1}, \gamma_{2}, \gamma_{3}, \gamma_{L}$, and $\gamma_{R}$ such that $g\left(\gamma_{1}\right)=g\left(\gamma_{3}\right)=1$; 
$g\left(\gamma_{2}\right)=g-2$; and there is a diagram (2.3):

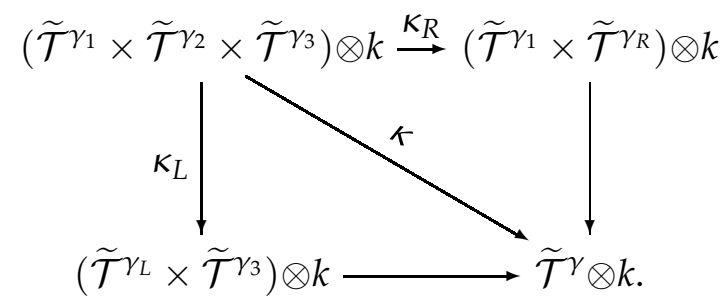

Let $\left(s_{1}, s_{2}, s_{3}\right) \in\left(\tilde{\mathcal{T}} \gamma_{1} \times \tilde{\mathcal{T}} \gamma_{2} \times \tilde{\mathcal{T}}^{\gamma_{3}}\right)(k)$, and let $s=\kappa\left(s_{1}, s_{2}, s_{3}\right)$. Let $V=\operatorname{Pic}^{0}\left(\mathcal{C}^{\gamma}\right)[\ell]_{s}$, and for $i=1,2,3$ let $V_{i}=\operatorname{Pic}^{0}\left(\mathcal{C}^{\gamma_{i}}\right)[\ell]_{s_{i}}$. Then $V \cong V_{1} \oplus V_{2} \oplus V_{3}$ is a decomposition of $V$ as a Hermitian $\left(\mathbb{Z}\left[\zeta_{3}\right] \otimes k\right)$-module (Lemma $\left.3.1(a)\right)$. Let $\widetilde{\mathcal{B}}_{R}$ be the image of $\kappa_{R}$, and let $\widetilde{\mathcal{B}}_{L}$ be the image of $\kappa_{L}$. Lemma 3.1(b) shows that $\mathrm{M}_{\ell}(\widetilde{\mathcal{T}} \gamma \otimes k, s)$ contains $\mathrm{M}_{\ell}\left(\widetilde{\mathcal{B}}_{L} \otimes k\right)$ and $\mathrm{M}_{\ell}\left(\widetilde{\mathcal{B}}_{R} \otimes k\right)$; the inductive hypothesis shows that these are $\mathrm{SU}\left(V_{1} \oplus V_{2}\right)$ and $\mathrm{SU}\left(V_{2} \oplus V_{3}\right)$, respectively.

If $\ell$ is inert in $\mathbb{Z}\left[\zeta_{3}\right]$, Lemma 3.2 $\mathrm{b}$ (ii) implies that $\mathrm{M}_{\ell}\left(\widetilde{\mathcal{T}}^{\gamma} \otimes k\right) \cong \mathrm{SU}_{g}\left(\mathbb{F}_{\ell^{2}}\right) \cong \mathrm{SG}_{\left(r_{\gamma}, s_{\gamma}\right)}(\mathbb{Z} / \ell)$.

Otherwise, if $\ell=\lambda \cdot \bar{\lambda}$ is split in $\mathbb{Z}\left[\zeta_{3}\right]$, let $V(\lambda)$ be the eigenspace of $V$ corresponding to $\lambda$, and define $V_{i}(\lambda)$ analogously for $i=1,2,3$. In this case, $\mathrm{SG}_{\left(r_{\gamma}, s_{\gamma}\right)}(\mathbb{Z} / \ell), \operatorname{SU}(V)$ and $\mathrm{SL}(V(\lambda))$ are isomorphic. By the inductive hypothesis, the projection of $\mathrm{M}_{\ell}\left(\tilde{\mathcal{T}}^{\gamma} \otimes k\right)$ to $\mathrm{SL}(V(\lambda))$ contains $\mathrm{SL}\left(V_{1}(\lambda) \oplus V_{2}(\lambda)\right)$ and $\mathrm{SL}\left(V_{2}(\lambda) \oplus V_{3}(\lambda)\right)$. By Lemma 3.2(a), we see that $\mathrm{M}_{\ell}\left(\widetilde{\mathcal{T}}^{\gamma} \otimes k\right) \cong \mathrm{SL}(V(\lambda)) \cong \mathrm{SG}_{\left(r_{\gamma}, s_{\gamma}\right)}(\mathbb{Z} / \ell)$.

Corollary 3.10. Let $\ell$ be an odd prime, and let $k$ be an algebraically closed field in which $3 \ell$ is invertible. Let $\widetilde{\mathcal{T}}^{\gamma}$ be any component of the moduli space of trielliptic curves. If $g(\gamma) \geq 3$, then $\mathrm{M}_{\mathbb{Z}_{\ell}}\left(\widetilde{\mathcal{T}} \gamma_{\otimes k} \cong \mathrm{M}_{\mathbb{Z}_{\ell}}\left(\mathcal{T}^{\gamma} \otimes k\right) \cong \mathrm{SG}_{\left(r_{\gamma}, s_{\gamma}\right)}\left(\mathbb{Z}_{\ell}\right)\right.$

Proof. The proof is parallel to that of Corollary 3.5; any subgroup of $\mathrm{SG}_{\left(r_{\gamma}, s_{\gamma}\right)}\left(\mathbb{Z}_{\ell}\right)$ which surjects onto $\mathrm{SG}_{\left(r_{\gamma}, s_{\gamma}\right)}(\mathbb{Z} / \ell)$ is all of $\mathrm{SG}_{\left(r_{\gamma}, s_{\gamma}\right)}\left(\mathbb{Z}_{\ell}\right)$.

Corollary 3.11. Let $p \neq 3$. Let $g \geq 3$ and let $(r, s)$ be a trielliptic signature for $g$ (Definition 2.9]. Then there exists a smooth trielliptic curve defined over $\overline{\mathbb{F}}_{p}$ with genus $g$ and signature $(r, s)$ whose Jacobian is absolutely simple.

Proof. Let $\mathbb{F}=\mathbb{F}_{p^{2}}$. Let $\ell$ be an odd prime distinct from $p$ which is inert in $\mathbb{Z}\left[\zeta_{3}\right]$, and let $K_{\ell}=\mathbb{Q}_{\ell}\left(\zeta_{3}\right)$. By Lemma 2.8 there is a class vector $\gamma$ whose inertia type has signature $(r, s)$. As in the proof of Corollary 3.6, using Corollary 3.10 one sees that $\mathrm{M}_{\mathbb{Q}_{\ell}}\left(\widetilde{\mathcal{T}}^{\gamma} \otimes \mathbb{F}\right) \cong$ $\mathrm{GU}_{(r, s)}\left(K_{\ell}\right)$.

Let $F$ be a $C M$ field of degree $[F: \mathbb{Q}]=2 g$ which contains $\mathbb{Q}\left(\zeta_{3}\right)$ and is inert at $\ell$, and let $F_{\ell}=F \otimes \mathbb{Q}_{\ell}$. There is a torus $H \subset \mathrm{GU}_{(r, s)}\left(K_{\ell}\right)$ isomorphic to $F_{\ell}^{\times}$. This torus is maximal since $[F: \mathbb{Q}]=2 g$. The quotient of $H$ by the center of $\mathrm{GU}_{(r, s)}\left(K_{\ell}\right)$ is isogenous to the kernel $J$ of the norm map $F_{\ell}^{\times} \rightarrow \mathbb{Q}_{\ell}^{\times}$. Since $F_{\ell}$ is a field, $J$ is anisotropic and $H$ is elliptic. Finally, $H$ acts irreducibly on the Tate module of the Jacobian of the tautological curve $C^{\gamma}$, since $T_{\ell}\left(\operatorname{Pic}^{0}\left(\mathcal{C}^{\gamma}\right)\right) \otimes \mathbb{Q}_{\ell}$ is a one-dimensional $F_{\ell}$-vector space. The result then follows from [6, Remark 5(i)]. 


\section{References}

[1] N. A'Campo. Tresses, monodromie et le groupe symplectique. Comment. Math. Helv., 54(2):318-327, 1979.

[2] J. D. Achter. The distribution of class groups of function fields. J. Pure Appl. Algebra, 204(2):316-333, 2006.

[3] J. Bellä̈che. Relèvement des formes modulaires de Picard. J. London Math. Soc., 74(1):13-25, 2006.

[4] S. Bosch, W. Lütkebohmert, and M. Raynaud. Néron models, volume 21 of Ergebnisse der Mathematik und ihrer Grenzgebiete (3) [Results in Mathematics and Related Areas (3)]. Springer-Verlag, Berlin, 1990.

[5] R. W. Carter. Finite groups of Lie type. Wiley Classics Library. John Wiley \& Sons Ltd., Chichester, 1993. Conjugacy classes and complex characters, Reprint of the 1985 original, A Wiley-Interscience Publication.

[6] C.-L. Chai and F. Oort. A note on the existence of absolutely simple Jacobians. J. Pure Appl. Algebra, 155(2-3):115-120, 2001.

[7] N. Chavdarov. The generic irreducibility of the numerator of the zeta function in a family of curves with large monodromy. Duke Math. J., 87(1):151-180, 1997.

[8] P. Deligne and D. Mumford. The irreducibility of the space of curves of given genus. Inst. Hautes Études Sci. Publ. Math., (36):75-109, 1969.

[9] T. Ekedahl. The action of monodromy on torsion points of Jacobians. In Arithmetic algebraic geometry (Texel, 1989), pages 41-49. Birkhäuser Boston, Boston, MA, 1991.

[10] T. Ekedahl. Boundary behaviour of Hurwitz schemes. In The moduli space of curves (Texel Island, 1994), volume 129 of Progr. Math., pages 173-198. Birkhäuser Boston, Boston, MA, 1995.

[11] G. Faltings and C.-L. Chai. Degeneration of abelian varieties. Springer-Verlag, Berlin, 1990. With an appendix by David Mumford.

[12] G. Frey, E. Kani, and H. Völklein. Curves with infinite K-rational geometric fundamental group. In Aspects of Galois theory (Gainesville, FL, 1996), volume 256 of London Math. Soc. Lecture Note Ser., pages 85-118. Cambridge Univ. Press, Cambridge, 1999.

[13] B. B. Gordon. Canonical models of Picard modular surfaces. In The zeta functions of Picard modular surfaces, pages 1-29. Univ. Montréal, Montreal, QC, 1992.

[14] E. W. Howe and H. J. Zhu. On the existence of absolutely simple abelian varieties of a given dimension over an arbitrary field. J. Number Theory, 92(1):139-163, 2002.

[15] N. M. Katz. Twisted L-functions and monodromy, volume 150 of Annals of Mathematics Studies. Princeton University Press, Princeton, NJ, 2002. 
[16] N. M. Katz and P. Sarnak. Random matrices, Frobenius eigenvalues, and monodromy. American Mathematical Society, Providence, RI, 1999.

[17] O. King. Maximal subgroups of the classical groups associated with nonisotropic subspaces of a vector space. J. Algebra, 73(2):350-375, 1981.

[18] F. F. Knudsen. The projectivity of the moduli space of stable curves. II. The stacks $M_{g, n}$. Math. Scand., 52(2):161-199, 1983.

[19] J. K. Koo. On holomorphic differentials of some algebraic function field of one variable over C. Bull. Austral. Math. Soc., 43(3):399-405, 1991.

[20] E. Kowalski. The large sieve, monodromy and zeta functions of curves, 2005. arXiv:math.NT/0503714.

[21] M. J. Larsen. Arithmetic compactification of some Shimura surfaces. In The zeta functions of Picard modular surfaces, pages 31-45. Univ. Montréal, Montreal, QC, 1992.

[22] M. J. Larsen. Maximality of Galois actions for compatible systems. Duke Math. J., 80(3):601-630, 1995.

[23] G. Laumon and L. Moret-Bailly. Champs algébriques, volume 39 of Ergebnisse der Mathematik und ihrer Grenzgebiete. 3. Folge. A Series of Modern Surveys in Mathematics [Results in Mathematics and Related Areas. 3rd Series. A Series of Modern Surveys in Mathematics]. Springer-Verlag, Berlin, 2000.

[24] B. Noohi. Fundamental groups of algebraic stacks. J. Inst. Math. Jussieu, 3(1):69-103, 2004.

[25] V. Platonov and A. Rapinchuk. Algebraic groups and number theory, volume 139 of Pure and Applied Mathematics. Academic Press Inc., Boston, MA, 1994.

[26] A. Vasiu. Surjectivity criteria for p-adic representations. I. Manuscripta Math., 112(3):325-355, 2003.

[27] H. Völklein. Groups as Galois groups, volume 53 of Cambridge Studies in Advanced Mathematics. Cambridge University Press, Cambridge, 1996. An introduction.

[28] Y. G. Zarhin. Cyclic covers of the projective line, their Jacobians and endomorphisms. J. Reine Angew. Math., 544:91-110, 2002. 